\title{
Explaining the dilatometric behavior during bainite transformation under the effect of variant selection
}

\author{
Adriana Eres-Castellanos ${ }^{\mathrm{a}, \mathrm{b}}$, Lucia Morales-Rivas ${ }^{\mathrm{b},}$, Francisca G. Caballero ${ }^{\mathrm{a}}$, Carlos Garcia- \\ Mateo $^{\mathrm{a}}$ \\ a Department of Physical Metallurgy, National Center for Metallurgical Research (CENIM-CSIC), Avda. Gregorio del Amo 8, \\ Madrid E-28040, Spain \\ ${ }^{\text {b}}$ Technische Universität Kaiserslautern (TUK), Materials Testing, Gottlieb-Daimler-Str., Kaiserslautern D-67663, Germany \\ *Correspondence: rivas@mv.uni-kl.de
}

\begin{abstract}
The selection of crystallographic variants in bainitic microstructures is a deeply studied topic which can be attributed to several reasons. In the cases where the prior austenite is textured, stressed or plastically deformed, the final ferrite texture and the dilatometry signal may change, being anisotropic. Although the causality between variant selection and the variation of the dilatometric signal has been previously assumed, it has not been thoroughly proved. In this work, the phenomenological theory of martensite was combined with a continuum mechanics analysis to calculate the relative length changes associated to the bainitic transformation during different bainitic transformations in order to prove the connection between the selection of crystallographic variants and the distortion of the dilatometry signal. To do so, four different bainitic microstructures were analyzed: one of them obtained by a pure isothermal treatment and the rest of them obtained by ausforming treatments. Some of the mentioned microstructures had shown to lead to negative longitudinal dilatometry signals and to present a strong variant selection. Results showed that the experimental trends could be explained by theoretical means, which enables to further understand the bainitic transformation.
\end{abstract}

Keywords: bainite, ausforming, variant selection, phenomenological theory of martensite, dilatometry

\section{Introduction}

Bainitic transformations are allotropic transformations that have been defined as displacive, as both transformations occur by homogeneously deforming the original pattern into a new crystal structure [1]. The formed ferrite keeps the orientation relationship (OR) with their corresponding parent austenite, being this OR defined by the parallelism of a plane and a 
direction of the parent austenite and a plane and a direction of the formed ferrite, respectively. Due to the lattice symmetry, there are usually 3, 12 or 24 different resultant orientations that can be formed as the result of a bainitic transformation in a single parent grain, the so-called crystallographic variants. Some of the most known OR are the Bain OR (B-OR), i.e. $<0.00$ $0.001 .00>45^{\circ}[2]$, the Nishiyama-Wassermann OR (NW-OR), i.e. $<0.200 .080 .98>45.98$ $\circ[3,4]$, the Kurdjumov-Sachs OR (KS-OR), i.e. $<0.180 .180 .97>42.85^{\circ}[5]$, or the Greninger-Troiano OR (GT-OR), i.e. $<0.130 .190 .97>44.23^{\circ}$ [6], among others [7,8]. However, in some cases, not all the possible variants are formed within a prior austenite grain. This phenomenon is called variant selection and it can be due to many different reasons, including: a) the prior austenite grain size (PAGS) is smaller than a few micrometers [9-12], b) the prior austenite is textured [13]; c) the prior austenite is stressed [14,15] or plastically deformed [16-19]; d) the driving force for the transformation is low in absolute value [13] so, either the transformation temperature is low [13], the carbon content is high [20] or the concentration of the austenite stabilizer substitutional elements is relatively high [13]. Nevertheless, not all these cases can be detected at a macroscopic level. When variant selection is related to small prior austenite grains or low driving forces, variants are not selected based on their relative orientation with respect to the sample coordinate system, but arbitrarily. Therefore, the final ferrite texture is not altered with respect to the one that would be expected if no variant selection happened and the dilatometry signal is not altered either. When variant selection occurs because the prior austenite is textured, stressed or plastically deformed, variants are selected based on their orientation with respect to the sample coordinate system and, thus, the final ferrite texture and the dilatometry signal may change with respect to the expected ones, becoming the dilatometry signal anisotropic - i.e. the longitudinal signal is not the same than the transverse one - and even negative along specific directions in some occurrences [19,21-23]

Although the causality between variant selection and the variation of the dilatometric signal has been previously assumed [21,22], it has not been thoroughly proved. He et al. [24] tried to explain these phenomena, although they only analyzed 11 prior austenite grains and, even despite they claimed that they had applied the Phenomenological Theory of Martensite (PTM) for their analysis, they skipped the consideration of the shape change on which the PTM is based. For that reason, in this work, the PTM was combined with a continuum mechanics analysis to study the relationship between variant selection and an anisotropic dilatometry signal. To do so, a medium carbon (0.4 wt. \%) high silicon (3.0 wt. \%) steel (Sidenor's SCM40), was used. Four different bainitic microstructures were analyzed: one of them obtained by a pure isothermal treatment and the rest of them obtained by ausforming treatments. Some of the mentioned microstructures had previously shown to lead to negative longitudinal dilatometry signals [22] and a strong variant selection phenomenon [19]. A theoretical methodology was applied to calculate the relative length changes associated to the bainitic transformation in those four different samples in order to prove the connection between the selection of crystallographic variants and the distortion of the dilatometry signal.

\section{Experimental}

In this work, a medium carbon (0.4 wt. \%) high silicon (3.0 wt. \%) steel (Sidenor's SCM40), was used. This steel also contained other alloying elements to control the hardenability. 
The technique of dilatometry was used to subject the steel to different thermal and thermomechanical treatments. To do so, a Bahr 805D high-resolution dilatometer was used. This dilatometer had an induction heating coil, which enabled to increase the temperature, and a helium quench system to decrease it. The temperature was controlled by a type K thermocouple, which was welded to the central part of the sample surface. The dilatometer can be equipped with two different linear variable displacement transducer (LVDT) modules, depending on whether deformation is applied during the treatment or not. Both LVDT modules enable to measure longitudinal displacements which can be associated to phase transformations. For thermal treatments, the LVDT module consists of fused silica push-rods, which have a very low thermal expansion coefficient $\left(\sim 0.510^{-6}{ }^{\circ} \mathrm{C}^{-1}\right)$ in comparison with the one of steel $\left(\sim 1010^{-6}{ }^{\circ} \mathrm{C}^{-1}\right)$. These push-rods are very accurate. However, for thermomechanical treatments, the module consists of silicon nitride punchers which also act as push-rods. The thermal expansion coefficient of silicon nitride is much higher than the one of fused silica $\left(\sim 3.310^{-6}{ }^{\circ} \mathrm{C}^{-1}\right)$, which impairs the accuracy of the dilatometer. The punchers are separated from the samples by molybdenum films to reduce friction and avoid barreling as much as possible.

Dilatometry samples of 4 and $5 \mathrm{~mm}$ diameter and $10 \mathrm{~mm}$ length were subjected to several thermal and thermomechanical treatments, whose sketches are included in Figure 1. In all cases, samples were austenitized at $990{ }^{\circ} \mathrm{C}$ for $4 \mathrm{~min}$. The austenitization temperature was proved to be well above the Ac3 $\left(930^{\circ} \mathrm{C}\right)$. From this point on, the treatment changed depending on the sample. One of the samples was subjected to a pure isothermal treatment. For that purpose, the sample was cooled down to $300{ }^{\circ} \mathrm{C}$ and held at such temperature for 1 $\mathrm{h}$. Note that this isothermal temperature was in the range between the martensite start temperature (Ms) and the bainite start temperature (Bs), i.e. between 280 and $499{ }^{\circ} \mathrm{C}$, according to Ref. [22]. Finally, it was cooled down to room temperature. The remaining three samples were subjected to ausforming treatments, which included a cooling step from $990{ }^{\circ} \mathrm{C}$ down to the deformation temperature $\mathrm{T}_{\text {def }}\left(\mathrm{T}_{\text {def }}=520,400\right.$ and $\left.300{ }^{\circ} \mathrm{C}\right)$. Then, $\mathrm{T}_{\text {def }}$ was held for $15 \mathrm{~s}$ before samples were compressed up to $10 \%$ at $0.04 \mathrm{~s}^{-1}$. In previous works in this same steel and with the same austenitization and deformation conditions, it was shown that, although some stress/strain induced bainite/martensite formed during this deformation step [25], the presence of these phases did not alter the trends observed in terms of variant selection with respect to samples in which no strain induced transformations were detected [19] and, thus, their presence could be neglected. After the stress was removed, samples were held at $\mathrm{T}_{\text {def }}$ for $10 \mathrm{~s}$ and cooled down to $300{ }^{\circ} \mathrm{C}$, temperature which was held for $1 \mathrm{~h}$ before cooling down to room temperature. The only phase transformation that was detected during these heat treatments, excepting the formation of traces of stress/strain induced bainite/martensite previously described, occurred during the isothermal step and was bainite. For the purpose of this work, it is relevant to note that no martensitic transformation was detected on cooling to room temperature. For further details on the selection of the heat treatment parameters, please refer to Ref. [22]. 


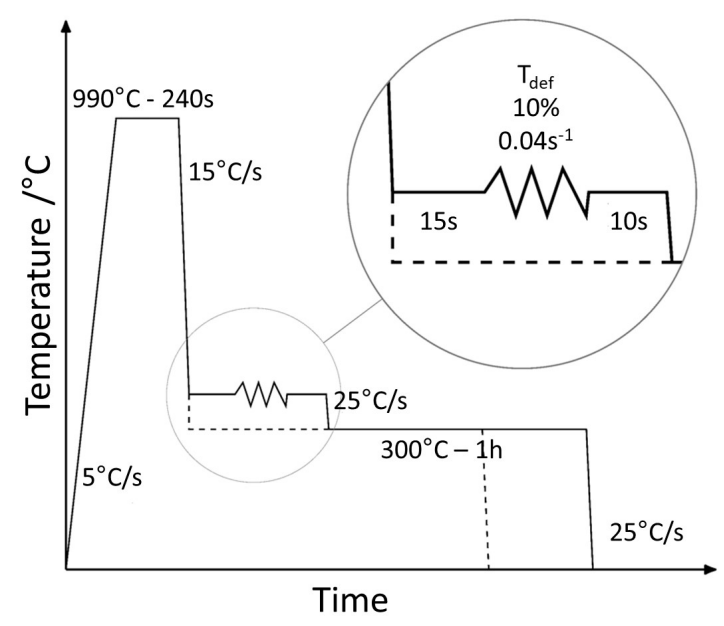

Figure 1. Sketch of the thermal (dashed line) and thermomechanical (solid line) treatments performed in Ref. [22] and replicated in this work. $T_{\text {def }}$ stands for deformation temperature.

To quantify the bainitic ferrite and retained austenite volume fractions of the obtained microstructures, samples were step scanned by a Bruker AXS D8 X-Ray diffractometer. XRay Diffraction (XRD) data was collected for a $2 \theta$ range of $35-135^{\circ}$ with a step size of 0.01 $\circ$. Ferrite volume fractions were estimated by integrating the intensities under the $\left(\begin{array}{lll}2 & 0 & 0\end{array}\right)$, (2 11 ) and (2 20 ) peaks. Similarly, austenite volume fractions were estimated by considering the $\left(\begin{array}{lll}2 & 0 & 0\end{array}\right),\left(\begin{array}{lll}2 & 2 & 0\end{array}\right)$ and $\left(\begin{array}{lll}3 & 1 & 1\end{array}\right)$ peaks. Also, this equipment was used to carry out global texture measurements by obtaining the following incomplete pole figures (PF) in the back reflection mode: (2 00 ), (2 11 1) and (2 200$)$ for the bainitic ferrite and (2 00 ), (2 200 ) and (3 111 ) for the retained austenite. The pole distance was varied from 0 to $70^{\circ}$ with a step size of $5^{\circ}$. The Orientation Distribution Function (ODF) was derived from these PF by using the Bunge's series expansion method [26] ( $1 \max$ of 22 or 14). The ODF was finally ghost corrected.

A Zeiss Auriga Compact FIB-SEM, operating at $20 \mathrm{kV}$, was used to carry out Electron Backscattered Diffraction (EBSD) measurements. $230 \times 173 \mu \mathrm{m}^{2}$ areas were scanned on the transverse section. A step size of $0.35 \mu \mathrm{m}$ was utilized and only the ferrite (bcc) structure was considered. The XRD data and the EBSD data had also been used in two previous works $[19,22]$ in which through characterizations was performed.

Samples were metallographically prepared for EBSD and XRD by using standard metallographic procedures, followed by etching and polishing cycles. These cycles are useful to remove any stress/strain induced martensite formed during the grinding step. Finally, samples were polished with a $50 \mathrm{~nm}$ colloidal silica suspension.

The subsequent analysis of the XRD and the EBSD data was performed by means of MTEX [27], a Matlab ${ }^{\circledR}$ toolbox. The reconstruction of the prior austenite was performed by the software by Nyyssönen et al. [28]. The algorithm on which this software is based consists of three steps: determination of the OR from intergranular misorientations, determination of areas which share the same ferrite orientation and their neighbors and separation of discrete clusters. More information about this algorithm can be found in Ref. [28]. 


\section{Theory and calculations}

This section deals with the calculation of the volume changes and length changes associated to a bainitic transformation, besides some important features that characterize the bainitic transformations.

Bainitic transformations imply that a fcc crystal $-\gamma-$ transforms to a bcc crystal $-\alpha-$ through a displacive transformation. Because the OR was estimated by software that does not consider tetragonality, as later explained, tetragonality will not be considered in the present work.

According to the theory by Bhadeshia [13], no diffusion occurs during the growth of a bainitic platelet, although carbon partitions from $\alpha$ to $\gamma$ right after the platelet growth is finished. Hence, one can define the bainitic total relative volume change $\left(R V C_{\text {total }}^{\text {bainite }}\right)$ as the summation of $R V C_{\text {crystal }}$ and a second term describing the relative volume change due to the carbon partition, $R V C_{\text {partition }}^{\text {bainite }}$, see Equation (1).

$$
R V C_{\text {total }}^{\text {bainite }}=R V C_{\text {crystal }}+R V C_{\text {partition }}^{\text {bainite }}
$$

Although the carbon enrichment of the matrix should be considered for the calculation of the mentioned volume changes, i.e. Equation (1) should be applied to calculate the values of

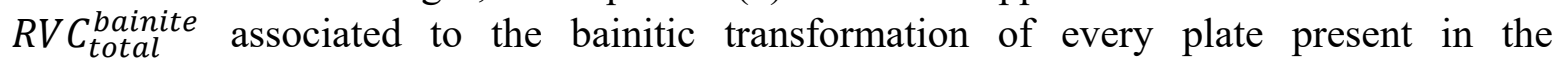
microstructure, in this work, because of the complexity of the calculation, it has been assumed that partition happens after all the bainitic ferrite plates have grown. Although this way could lead to errors, it helps to simplify the calculation and not to have to make other assumptions, such as the volume fraction of every platelet, the variation of the lattice parameters as the transformation progresses or the variation of the OR.

Concerning the changes in length, at this point it is noteworthy to point out that two different scales will be regarded: length changes will be calculated for a single bainitic lath/plate (local scale) and for a whole sample (global scale). This distinction is necessary because, while the volume change given by the compositional change during partition is isotropic even for a single lath/plate - i.e. the length changes accompanying the carbon partition of a single lath/plate towards the austenite that surrounds it are always the same along all directions -, the volume change given by the crystallographic change is not. Because of the shear nature of the bainitic transformation, this crystallographic volume change is anisotropic at the local scale, so the length changes associated to the transformation of a single lath/plate are never the same along all directions. The crystallographic volume change is isotropic at the global scale only if no variant selection phenomena are shown. In that case, the local relative volume changes and the relative length changes of all laths/plates average out. Equation (2) shows an approximate equality describing the relative length change ( $R L C)$ accompanying any isotropic relative volume change $(R V C)$ [29].

$$
R L C \approx \frac{R V C}{3}
$$


When variant selection phenomena are found, the calculation of the global relative length changes accompanying a bainitic transformation is not so straightforward and a deeper analysis is required.

In the next subsections, it will be shown how to calculate the total global relative length changes associated to a bainitic transformation, regardless of whether the transformation is globally anisotropic or not. To do so, first, it will be assessed how to calculate the isotropic relative length changes associated to the carbon partition after bainitic growth. Second, the PTM will be combined with a continuum mechanics analysis to show the theory behind the calculation of the relative length changes associated to the crystal structure change.

\subsection{Volume and length changes associated to carbon partition during bainitic transformations}

As mentioned, the partition of carbon during bainite reaction is linked to an isotropic relative volume change $R V C_{\text {partition, }}^{\text {bainite }}$, which is given by the density difference between $\gamma$ and $\alpha$. To calculate it, let us imagine a $\gamma$ crystal with $N$ atoms, whose lattice parameter (before partition) is $a_{\gamma, B P}$ and whose volume is $V_{\text {initial }}$, as defined in Equation (3):

$$
V_{\text {initial }}=\frac{N}{n_{\gamma}} a_{\gamma, B P}^{3}
$$

where $n_{\gamma}$ is the number of atoms of the $\gamma$ cell, i.e. $n_{\gamma}=4$. A fraction $F_{\alpha}$ of the crystal transforms to $\alpha$, remaining a volume fraction $F_{\gamma}=1-F_{\alpha}$ untransformed. At this point, i.e. before partition, the $\alpha$ lattice parameter is $a_{\alpha, B P}$ and the total volume is $V_{B P}$, see Equation (4) for its formula.

$$
V_{B P}=\frac{N}{n_{\gamma}} a_{\gamma, B P}^{3} F_{\gamma}+\frac{N}{n_{\alpha}} a_{\alpha, B P}^{3} F_{\alpha}
$$

where $n_{\alpha}$ is the number of atoms of the $\alpha$ cell, i.e. $n_{\alpha}=2$. After growth is finished, partition occurs and the lattice parameters (after partition) of $\gamma$ and $\alpha$ are $a_{\gamma, A P}$ and $a_{\alpha, A P}$ now, respectively. Therefore, after partition, the total volume $V_{A P}$ is:

$$
V_{A P}=\frac{N}{n_{\gamma}} a_{\gamma, A P}^{3} F_{\gamma}+\frac{N}{n_{\alpha}} a_{\alpha, A P}^{3} F_{\alpha}
$$

The $R V C_{\text {partition }}^{\text {bainite }}$ is defined as the volume change related to carbon partition with respect to the initial volume and can be calculated as shown in Equation (6).

$$
R V C_{\text {partition }}^{\text {bainite }}=\frac{V_{A P}-V_{B P}}{V_{\text {initial }}}=\frac{\left(a_{\gamma, A P}^{3}-a_{\gamma, B P}^{3}\right)}{a_{\gamma, B P}^{3}} F_{\gamma}+\frac{n_{\gamma}\left(a_{\alpha, A P}^{3}-a_{\alpha, B P}^{3}\right)}{n_{\alpha} a_{\gamma, B P}^{3}} F_{\alpha}
$$


Because partition occurs isotropically, the partition relative length change $\left(R L C_{\text {partition }}^{\text {bainite }}\right)$ can be approximately calculated as follows:

$$
R L C_{\text {partition }}^{\text {bainite }} \approx \frac{R V C_{\text {partition }}}{3}
$$

\subsection{Volume and length changes associated to the crystal structure change during bainitic transformations}

Let us imagine the same scenario described in Section 3.1, where a fraction $F_{\alpha}$ of a $\gamma$ crystal with $N$ atoms and a lattice parameter $a_{\gamma, B P}$ gets transformed to a $\alpha$ lattice with a lattice parameter $a_{\alpha, B P}$. The $R V C_{\text {crystal }}$ is defined as the volume change related to the crystal structure change with respect to the initial volume and can be calculated as shown in Equation (8).

$$
R V C_{\text {crystal }}=\frac{V_{B P}-V_{\text {initial }}}{V_{\text {initial }}}=\frac{2 a_{\alpha, B P}^{3}-a_{\gamma, B P}^{3}}{a_{\gamma, B P}^{3}} F_{\alpha}
$$

As previously explained, because the crystal structure change does not occur isotopically at the local scale, it is only when both global relative length changes are considered, and no variant selection phenomena are found, that the relative length change accompanying the crystal structure change $\left(R L C_{\text {crystal }}\right)$ can be approximately calculated as follows:

$$
R L C_{\text {crystal }} \approx \frac{R V C_{\text {crystal }}}{3}
$$

If that condition is not met or the determination of local relative length changes is aimed, Equation (9) is not valid and a further analysis, which includes the PTM and a continuum mechanics analysis, must be carried out.

For the sake of clarity, during the next subsections, it will be assumed that the $\gamma$ crystal of study - with an orientation $(\gamma \boldsymbol{J} R)$ with respect the sample reference frame $(R)$ - fully transforms to $\alpha\left(F_{\alpha}=1\right)$ and that the transformed $\alpha$ has a unique orientation, $(\alpha \boldsymbol{J} R)$. Finally, in the last subsection, it will be shown how to deal with more complex structures, where different $\gamma$ grains transform to $\alpha$ laths/plates with different orientations and where some $\gamma$ remains untransformed.

\subsubsection{Transformation strain}

Given the displacive character of the bainitic transformations [30] there is an associated deformation which can be described by the material deformation gradient tensor $(\gamma \boldsymbol{A} \gamma)$. This deformation gradient tensor can be used to calculate the vector describing the final position of a point, $[\gamma ; \boldsymbol{x}]$, by knowing its initial position, characterized by a position vector $[\gamma ; \boldsymbol{X}]$, see Equation (10).

$$
[\gamma ; \boldsymbol{x}]=(\gamma \boldsymbol{A} \gamma)[\gamma ; \boldsymbol{X}]
$$


This transformation deformation gradient tensor, which is anisotropic and which is known to be an invariant-line deformation (ILD) [31], can be calculated by Equation (11) [32]. In this equation, the Bain deformation gradient tensor $(\gamma \boldsymbol{B} \gamma)$ describes the homogeneous deformation required for the $\gamma$ crystal structure to become a $\alpha$ crystal structure [2], and it is defined by Equation (12). As can be observed, such homogeneous deformation consists of a compression along one axis (as $a_{\alpha, B P}<a_{\gamma, B P}$ ) and an expansion along the other two axes. In order for $(\gamma \boldsymbol{A} \gamma)$ to lead to an ILD, a rigid body rotation $(\gamma \boldsymbol{R} \gamma)$ of only few degrees must be applied to $(\gamma \boldsymbol{B} \gamma)$.

$$
\begin{gathered}
(\gamma \boldsymbol{A} \gamma)=(\gamma \boldsymbol{R} \gamma)(\gamma \boldsymbol{B} \gamma) \\
(\gamma \boldsymbol{B} \gamma)=\left(\begin{array}{ccc}
\sqrt{2} \frac{a_{\alpha, B P}}{a_{\gamma, B P}} & 0 & 0 \\
0 & \sqrt{2} \frac{a_{\alpha, B P}}{a_{\gamma, B P}} & 0 \\
0 & 0 & \frac{a_{\alpha, B P}}{a_{\gamma, B P}}
\end{array}\right)
\end{gathered}
$$

Note that, because $(\gamma \boldsymbol{A} \gamma)$ is a deformation gradient tensor, its determinant equals the ratio of the deformed to the undeformed volume elements, see Equation (13).

$$
R V C_{\text {crystal }}=(|(\gamma \boldsymbol{A} \gamma)|-1) F_{\alpha}
$$

The deformation gradient tensor can also be derived from the coordinate transformation matrix representing the orientation relationship between $\alpha$ and $\gamma,(\gamma J \alpha)$, and the Bain correspondence matrix, ( $\alpha \boldsymbol{C} \gamma)$, as shown in Equation (14) [32].

$$
(\gamma \boldsymbol{A} \gamma)=\frac{a_{\alpha}}{a_{\gamma}}(\gamma \boldsymbol{J} \alpha)(\alpha \boldsymbol{C} \gamma)
$$

For a known $(\gamma \boldsymbol{J} \alpha)$ and a known austenite crystal orientation $(\gamma \boldsymbol{J} R)$, the formed ferrite crystal orientation $(\alpha \boldsymbol{J} R)$ can be calculated by using Equation (15) [32].

$$
(\alpha \boldsymbol{J} R)=(\alpha \boldsymbol{J} \gamma)(\gamma \boldsymbol{J} R)
$$

Note that $(\alpha \boldsymbol{J} \gamma)=(\gamma \boldsymbol{J} \alpha)^{\mathbf{- 1}}$. Concerning $(\alpha \boldsymbol{C} \gamma)$, its columns are rational lattice vectors describing the $\gamma$ basis vectors in the $\alpha$ basis, e.g. matrix shown in Equation (16) [32].

$$
(\alpha C \gamma)=\left(\begin{array}{lll}
1 & \overline{1} & 0 \\
1 & 1 & 0 \\
0 & 0 & 1
\end{array}\right)
$$

Because of the symmetry presented by the crystal structures, there are 48 symmetrically equivalent $(\alpha \boldsymbol{C} \gamma)$. The matrix $(\alpha \boldsymbol{C} \gamma)$ which leads to the lowest rigid body rotation $(\gamma \boldsymbol{R} \gamma)$ in Equation (11) must be chosen. 


\subsubsection{Invariant line determination}

As previously said, the bainitic transformation is characterized by being an ILD, i.e. the deformation associated to the bainitic transformation must leave a line undeformed: the socalled invariant line (IL) [31]. In this subsection, it will be assessed how to calculate the orientation of such a line.

To do so, some concepts must be firstly described. The spatial displacement vector, $[\gamma ; \boldsymbol{\Delta x}]$, of a point whose initial and final position vectors are $[\gamma ; \boldsymbol{X}]$ and $[\gamma ; \boldsymbol{x}]$, respectively, can be obtained by using Equation (17):

$$
[\gamma ; \boldsymbol{\Delta x}]=[\gamma ; \boldsymbol{x}]-[\gamma ; \boldsymbol{X}]=\left(\gamma \boldsymbol{t}_{\boldsymbol{A}} \gamma\right)[\gamma ; \boldsymbol{x}]
$$

$\left(\gamma \boldsymbol{t}_{\boldsymbol{A}} \gamma\right)$ is the spatial displacement gradient tensor, defined by Equation (18), where $\boldsymbol{I}$ is the identity matrix and $t_{i j}$ is the element in the $i^{\text {th }}$ row and $j^{\text {th }}$ column of $(\gamma \boldsymbol{t} \gamma)$.

$$
\left(\gamma \boldsymbol{t}_{\boldsymbol{A}} \gamma\right)=\left(\begin{array}{lll}
t_{11} & t_{12} & t_{13} \\
t_{21} & t_{22} & t_{23} \\
t_{31} & t_{32} & t_{33}
\end{array}\right)=\boldsymbol{I}-(\gamma \boldsymbol{A} \gamma)^{-1}
$$

These equations can be used to obtain the orientation of the IL. Such line is defined by an infinite number of position vectors, all of them pointing at the same direction, and whose spatial displacement vectors are all $\left[\gamma ; \Delta x_{I L}\right]=\left[\begin{array}{lll}0 & 0 & 0\end{array}\right]^{T}$.

The mentioned IL can be obtained analytically, as described in Appendix B, as long as the rank of $\left(\gamma \boldsymbol{t}_{\boldsymbol{A}} \gamma\right)$ is 2 . However, in the practice, when experimental results are used, it is very difficult to work with matrices $\left(\gamma \boldsymbol{t}_{\boldsymbol{A}} \gamma\right)$ whose rank is 2 . An alternative is to look for the line whose displacement is the closest to zero, whose unit vector will be called from now on experimental invariant line, $\left[\gamma ; \boldsymbol{\Delta} \boldsymbol{x}_{\boldsymbol{e x p}-\boldsymbol{I L}}\right]$. Applying the Singular Value Decomposition (SVD) to $\left(\gamma \boldsymbol{t}_{\boldsymbol{A}} \gamma\right)$ is very useful for such a purpose. A thorough explanation of the SVD and its geometrical meaning can be found in Appendix C. The SVD of $(\gamma t \gamma)$ is described in Equation (19).

$$
\left(\gamma \boldsymbol{t}_{\boldsymbol{A}} \gamma\right)=\left(\gamma \mathbf{u}_{\mathbf{t}} \gamma\right)\left(\gamma \boldsymbol{d}_{\boldsymbol{t}} \gamma\right)\left(\gamma \boldsymbol{v}_{\boldsymbol{t}} \gamma\right)^{T}
$$

The vector $\left[\beta ; \boldsymbol{u}_{t 3}\right]$, third column of $\left(\gamma \mathbf{u}_{\mathbf{t}} \gamma\right)$, shares the direction with the third principal deformation axis, being its corresponding principal deformation $\sigma_{3}$ (third diagonal value of $\left.\left(\gamma \boldsymbol{d}_{\boldsymbol{t}} \gamma\right)\right)$ minimal. Hence, $\left[\gamma ; \boldsymbol{\Delta} \boldsymbol{x}_{\boldsymbol{e x p}-\boldsymbol{I} \boldsymbol{L}}\right]=\sigma_{t 3}\left[\beta ; \boldsymbol{u}_{\boldsymbol{t} 3}\right]$ and $\left\|\left[\gamma ; \boldsymbol{\Delta} \boldsymbol{x}_{\boldsymbol{e x p}-\boldsymbol{I} \boldsymbol{L}}\right]\right\|=\sigma_{t 3}$. One of the final position vectors describing the IL, $\left[\gamma ; \boldsymbol{x}_{\boldsymbol{e x p}-I L}\right]$, can be calculated by substituting $[\gamma ; \boldsymbol{\Delta x}]$ for $\left[\gamma ; \boldsymbol{\Delta} \boldsymbol{x}_{\boldsymbol{e x p}-\boldsymbol{I L}}\right]$ in Equation (17). Note that, in case of the $(\gamma \boldsymbol{t} \gamma)$ rank being 2 , $\left[\gamma ; \boldsymbol{x}_{\text {exp-IL }}\right]=\left[\gamma ; \boldsymbol{x}_{I L}\right]$ and $\left[\gamma ; \boldsymbol{\Delta} \boldsymbol{x}_{\exp -I L}\right]=\left[\gamma ; \Delta \boldsymbol{x}_{I L}\right]=\left[\begin{array}{lll}0 & 0 & 0\end{array}\right]^{T}$.

Factorizing $(\gamma \boldsymbol{A} \gamma)$ by the SVD could also be useful to visualize this IL. To do so, the SVD of $(\gamma \boldsymbol{A} \gamma)$ must be carried out as described in Equation (20).

$$
(\gamma \boldsymbol{A} \gamma)=\left(\gamma \boldsymbol{U}_{\boldsymbol{A}} \gamma\right)\left(\gamma \boldsymbol{D}_{\boldsymbol{A}} \gamma\right)\left(\gamma \boldsymbol{V}_{\boldsymbol{A}} \gamma\right)^{T}
$$


The vectors $\left[\gamma ; \boldsymbol{U}_{\boldsymbol{A 1}}\right],\left[\gamma ; \boldsymbol{U}_{\boldsymbol{A 2}}\right]$ and $\left[\gamma ; \boldsymbol{U}_{\boldsymbol{A 3}}\right]$, columns of $\left(\gamma \boldsymbol{U}_{\boldsymbol{A}} \gamma\right)$, point at the principal deformation directions and the diagonal components of $\left(\gamma \boldsymbol{D}_{A} \gamma\right)-\sigma_{A 1}, \sigma_{A 2}$ and $\sigma_{A 3}$-are the principal deformation values. The values of $\sigma_{A 1}$ and $\sigma_{A 2}$ should be greater than the unity and equal, while $\sigma_{A 3}$ should be less than the unity. Remember that $(\gamma \boldsymbol{A} \gamma)$ is equivalent to the product of the Bain deformation tensor and a rotation tensor, the latter involving no deformation, and the Bain deformation consisting of a compression along one axis and an expansion along the remaining axes.

If a sphere of radius equal to the unity is drawn and a ellipsoid whose axes point at the mentioned principal deformation directions and are as long as the corresponding principal deformation values is superimposed on it, the effect of $(\gamma \boldsymbol{A} \gamma)$ can be observed. Besides, if $\left[\gamma ; \boldsymbol{X}_{\text {exp-IL }}\right]$ and $\left[\gamma ; \boldsymbol{x}_{\text {exp-IL }}\right]$ are included, it will be seen that both vectors are equal (or almost equal) and that they point the intersection of both the sphere and the ellipsoid. An example of this will be later shown in Section 4.

\subsubsection{Phenomenological theory of martensite (PTM)}

In Section 3.2.1, the deformation gradient tensor associated to the bainitic transformation $(\gamma \boldsymbol{A} \gamma)$ was defined, and it was also explained that it must be an ILD so that a displacive transformation can happen. This was concluded to be true as, in the literature, the experimentally observed $\alpha$ orientations were consistent with the ones predicted by $(\gamma \boldsymbol{A} \gamma)$ $[13,32]$. However, the experimentally observed shape deformation could not be described by $(\gamma \boldsymbol{A} \gamma)$ but by an invariant-plane deformation (IPD) [33], whose (shape) deformation gradient tensor will be called from now on $(\gamma \boldsymbol{P} \gamma)[13,32]$. The tensor $(\gamma \boldsymbol{P} \gamma)$, though, never leads to the observed $\alpha$ lattice structure unless it is combined with a homogeneous shear deformation gradient tensor $(\gamma \boldsymbol{Q} \gamma)$ to give $(\gamma \boldsymbol{A} \gamma)$, as stated in Equation (21).

$$
(\gamma \boldsymbol{A} \gamma)=(\gamma \boldsymbol{P} \gamma)(\gamma \boldsymbol{Q} \gamma)
$$

To resolve this incongruence, it was proposed that the effect of $(\gamma \boldsymbol{Q} \gamma)$ is cancelled macroscopically by an inhomogeneous lattice-invariant deformation, which may be slip or twinning $[13,32]$, where twinning is usually favored at low temperatures or when high strain rates are involved, although the reasons explaining why one mechanism is predominant over the other is not fully understood yet [34,35]. Note that, to make the effect of $(\gamma \boldsymbol{Q} \gamma)$ invisible on a macroscopic scale, the deformation gradient $(\gamma \boldsymbol{Q} \gamma)$ must be a simple shear and do not lead to any volume change, i.e. $|(\gamma \boldsymbol{Q} \gamma)|=1$. In this way, the volume ratio associated to the transformation deformation gradient equals the one associated with the shape deformation gradient: $|(\gamma \boldsymbol{A} \gamma)|=|(\gamma \boldsymbol{P} \gamma)|$. The volume ratio associated to the shape deformation gradient $(\gamma \boldsymbol{P} \gamma), R V C_{\text {crystal-P }}$ can be calculated as shown in Equation (22). Note that, according to the theory, $R V C_{\text {crystal }}=R V C_{\text {crystal }-P}$.

$$
R V C_{\text {crystal-P }}=(|(\gamma \boldsymbol{P} \gamma)|-1) F_{\alpha}
$$

Regarding ( $\gamma \boldsymbol{Q} \gamma$ ), it is important to remind, as previously pointed out in previous works [35], that the lattice invariant shear does not happen after the lattice change has occurred, but simultaneously at the interface. 
Once the nature of $(\gamma \boldsymbol{P} \gamma)$ and $(\gamma \boldsymbol{Q} \gamma)$ is defined, it is possible to define these two deformation gradients. The shape change $(\gamma \boldsymbol{P} \gamma)$ consists of a dilatation deformation and a shear deformation and can be defined as shown in Equation (23):

$$
(\gamma \boldsymbol{P} \gamma)=\boldsymbol{I}+m[\gamma ; \boldsymbol{d}](\boldsymbol{p} ; \gamma)
$$

where $m$ is the magnitude of the shape deformation, $(\boldsymbol{p} ; \gamma)$ is the unit normal to the $\gamma$ habit plane and $[\gamma ; \boldsymbol{d}]$ is the displacement vector. $(\gamma \boldsymbol{Q} \gamma)$, as mentioned, must be a simple shear and lead to no volume change so that $|(\gamma \boldsymbol{A} \gamma)|=|(\gamma \boldsymbol{P} \gamma)|$. Such deformation gradient $(\gamma \boldsymbol{Q} \gamma)$ is of magnitude $n$ on a plane with unit normal $(\boldsymbol{q} ; \gamma)$ and along a unit direction $[\gamma ; \boldsymbol{e}]:$

$$
(\gamma \boldsymbol{Q} \gamma)=\boldsymbol{I}+n[\gamma ; \boldsymbol{e}](\boldsymbol{q} ; \gamma)
$$

It is common to assume that the lattice invariant shear occurs in $\left\{\begin{array}{lll}1 & 0 & 1\end{array}\right\}_{\gamma}<\overline{1} 01>_{\gamma}$ [32]. In that case, $(\gamma \boldsymbol{Q} \gamma)$, which is cancelled out by this lattice invariant shear, must be a shear on $\left\{\begin{array}{lll}1 & 0 & 1\end{array}\right\}_{\gamma}<\overline{1} 01>_{\gamma}$. Because it is known that $\left[\gamma ; \boldsymbol{x}_{\boldsymbol{I L}}\right]$ lies at the intersection of the habit plane and the plane on which the lattice-invariant shear occurs [13], $(\boldsymbol{q} ; \gamma)$ must be selected so that its corresponding plane, belonging to the family $\left\{\begin{array}{lll}1 & 0 & 1\end{array}\right\}_{\gamma}$, contains $\left[\gamma ; \boldsymbol{x}_{I L}\right]$, i.e. the angle between $(\boldsymbol{q} ; \gamma)$ and $\left[\gamma ; \boldsymbol{x}_{\boldsymbol{I L}}\right]$ must be as close as possible to, $90^{\circ}$. Moreover, the direction $[\gamma ; \boldsymbol{e}]$ must also be chosen from the family $<\overline{1} 01>_{\gamma}$ so that it is contained in the selected plane, i.e. the angle between $(\boldsymbol{q} ; \gamma)$ and $[\gamma ; \boldsymbol{e}]$ must be as close as possible to, $90^{\circ}$.

Finally, to isolate $[\gamma ; \boldsymbol{d}]$ and $(\boldsymbol{p} ; \gamma)$, several operations must be applied. They are based on the definition of $(\gamma \boldsymbol{A} \gamma)$ shown in Equation (25) - obtained by substituting Equations (23) and (24) in Equation (21) - and on Equation (26), obtained by applying the inverse to Equation (25). Further information on the math can be found in Ref. [32].

$$
\begin{gathered}
(\gamma \boldsymbol{A} \gamma)=\{\boldsymbol{I}+m[\gamma ; \boldsymbol{d}](\boldsymbol{p} ; \gamma)\}\{\boldsymbol{I}+n[\gamma ; \boldsymbol{e}](\boldsymbol{q} ; \gamma)\} \\
(\gamma \boldsymbol{A} \gamma)^{-1}=\{I-n[\gamma ; \boldsymbol{e}](\boldsymbol{q} ; \gamma)\}\left\{I-\frac{1}{1+m(\boldsymbol{p} ; \gamma)[\gamma ; \boldsymbol{d}]} m[\gamma ; \boldsymbol{d}](\boldsymbol{p} ; \gamma)\right\}
\end{gathered}
$$

From Equations (25) and (26), one can obtain Equation (27) and (28), respectively [32].

$$
\begin{aligned}
& c m[\gamma ; \boldsymbol{d}]=(\gamma \boldsymbol{A} \gamma)[\gamma ; \boldsymbol{e}]-[\gamma ; \boldsymbol{e}] \\
& b(\boldsymbol{p} ; \gamma)=(\boldsymbol{q} ; \gamma)-(\boldsymbol{q} ; \gamma)(\gamma \boldsymbol{A} \gamma)^{-1}
\end{aligned}
$$

where $c$ and $b$ are scalar numbers given by Equations (29) and (30).

$$
\begin{gathered}
c=(\boldsymbol{p} ; \gamma)[\gamma ; \boldsymbol{e}] \\
b=\frac{1}{1+m(\boldsymbol{p} ; \gamma)[\gamma ; \boldsymbol{d}]} m(\boldsymbol{q} ; \gamma)[\gamma ; \boldsymbol{d}]
\end{gathered}
$$


Because $[\gamma ; \boldsymbol{d}]$ and $(\boldsymbol{p} ; \gamma)$ are unit vectors, the modulus of the right term of Equations (27) and (28) correspond to $\mathrm{cm}$ and $b$, respectively. Therefore:

$$
\begin{gathered}
m=\frac{\|(\gamma \boldsymbol{A} \gamma)[\gamma ; \boldsymbol{e}]-[\gamma ; \boldsymbol{e}]\|}{c} \\
b=\left\|(\boldsymbol{q} ; \gamma)-(\boldsymbol{q} ; \gamma)(\gamma \boldsymbol{A} \gamma)^{-1}\right\|
\end{gathered}
$$

Once $m$ and $b$ are known, $[\gamma ; \boldsymbol{d}]$ and $(\boldsymbol{p} ; \gamma)$ can be isolated from Equations (27) and (28) and $(\gamma \boldsymbol{P} \gamma)$ can be calculated by Equation (23). Subsequently, $(\gamma \boldsymbol{Q} \gamma)$ can be isolated from Equation (21). Moreover, the magnitude $m$ can be used to calculate the dilatational and shear components associated to the bainitic transformation $-\delta$ and $s$, respectively -, see Equations (33) and (34).

$$
\begin{aligned}
& \delta=m(\boldsymbol{p} ; \gamma)[\gamma ; \boldsymbol{d}] \\
& s=\left(m^{2}-\delta^{2}\right)^{1 / 2}
\end{aligned}
$$

The dilatational component $\delta$ has been experimentally shown to be $\sim 0.03$ [36-39]. Typical values of $s$ for bainitic transformations lie between 0.22 and 0.45 [37-40].

Additionally, the tensor $(\gamma \boldsymbol{P} \gamma)$ can be used to study the macroscopic deformation associated to the growth of bainite under adiffusional conditions along different directions by calculating the material displacement gradient tensor, $\left(\gamma \boldsymbol{T}_{\boldsymbol{P}} \gamma\right)$, as shown in Equation (35). Note that the basis of the tensor $\left(\gamma \boldsymbol{T}_{\boldsymbol{P}} \gamma\right)$ must be changed to the sample reference frame $R$, see Equation (36). The relative length changes along the $\mathrm{X}, \mathrm{Y}$ and $\mathrm{Z}$-axes are the principal components of $\left(\mathrm{R} \mathbf{T}_{\mathbf{P}} \mathrm{R}\right)$.

$$
\begin{gathered}
\left(\gamma \mathbf{T}_{\mathbf{P}} \gamma\right)=(\gamma P \gamma)-\boldsymbol{I} \\
\left(\mathbf{R} \mathbf{T}_{\mathbf{P}} \mathbf{R}\right)=(\mathbf{R} \boldsymbol{J} \gamma)\left(\gamma \mathbf{T}_{\mathbf{P}} \gamma\right)(\gamma \boldsymbol{J} R)
\end{gathered}
$$

Factorizing $(\gamma \boldsymbol{P} \gamma)$ by the SVD explained in Appendix C could also be useful to identify the axes along which the principal deformations occur. The SVD of $(\gamma \boldsymbol{P} \gamma)$ is described in Equation (37).

$$
(\gamma \boldsymbol{P} \gamma)=\left(\gamma \boldsymbol{U}_{\boldsymbol{P}} \gamma\right)\left(\gamma \boldsymbol{D}_{\boldsymbol{P}} \gamma\right)\left(\gamma \boldsymbol{V}_{\boldsymbol{P}} \gamma\right)^{T}
$$

The vectors $\left[\gamma ; \boldsymbol{U}_{\boldsymbol{P 1}}\right],\left[\gamma ; \boldsymbol{U}_{\boldsymbol{P 2}}\right]$ and $\left[\gamma ; \boldsymbol{U}_{\boldsymbol{P} 3}\right]$, columns of $\left(\gamma \boldsymbol{U}_{\boldsymbol{P}} \gamma\right)$, point the principal deformation directions and the diagonal components of $\left(\gamma \boldsymbol{D}_{\boldsymbol{P}} \gamma\right)-\sigma_{P 1}, \sigma_{P 2}$ and $\sigma_{P 3}-$ are the principal deformation values.

\subsubsection{Extrapolation to more complex structures}

The previous subsections focused on calculating the relative length changes accompanying the change of crystal structure from a single $\gamma$ crystal to a single $\alpha$ crystal, where no $\gamma$ remained untransformed. However, experimentally observed microstructures are more 
complex; they contain many different $\gamma$ grains which transform to many $\alpha$ laths/plates with different orientations and different sizes. Moreover, some $\gamma$ remains untransformed $[41,42]$.

In this work, it was assumed that the whole austenitic lattice transformed to bainitic ferrite and finally some correction factors were considered to deal with the fact that only a fraction of bainite was formed and that carbon partitioned. Note that, as previously discussed, it was assumed that partition took place after all the bainitic ferrite was formed. Therefore, the retained austenite was not considered and the bainitic ferrite was divided in blocks - areas with the same orientation -, where the orientation of the austenite from which those blocks transformed was known. The material displacement gradient tensor of the whole microstructure, ( $R \mathbf{T}_{\mathbf{P}, \mathbf{g l o b a l}} R$ ), could be then calculated by using Equation (38).

$$
\left(R \mathbf{T}_{\mathbf{P}, \text { global }} R\right)=\frac{F_{\alpha}}{\text { area }_{\text {total }}} \sum_{i=1}^{n} \operatorname{area}_{i}\left(R \mathbf{T}_{\mathbf{P . \mathbf { i }}} R\right)
$$

where $\left(R \mathbf{T}_{\mathbf{P}, \mathbf{i}} R\right)$ is the material displacement gradient tensor of the $i^{\text {th }} \alpha$ block, calculating according to Equation (36), are $a_{i}$ is the area of the $i^{\text {th }}$ block, are $a_{\text {total }}$ is the total area occupied by $\alpha$ blocks - area $_{\text {total }}=\sum_{i=1}^{n}$ area $_{i}$ - and $\mathrm{n}$ is the total number of $\alpha$ blocks.

The principal components of $\left(R \mathbf{T}_{\mathbf{P}, \text { global }} R\right), R L C_{\text {crystal-X }}, R L C_{\text {crystal-Y }}$ and $R L C_{\text {crystal }-Z}$, are the relative changes in length due to the crystal structure change along the $\mathrm{X}, \mathrm{Y}$ and $\mathrm{Z}$ axes, respectively, as described in Equation (39).

$$
\left(R \mathbf{T}_{\mathbf{P}, \text { global }} R\right)=\left(\begin{array}{ccc}
R L C_{\text {crystal }-X} & 0 & 0 \\
0 & R L C_{\text {crystal }-Y} & 0 \\
0 & 0 & R L C_{\text {crystal }-Z}
\end{array}\right)
$$

Note that, in case that no variant selection phenomena are found, $R L C_{\text {crystal }-X}=$ $R L C_{\text {crystal }-Y}=R L C_{\text {crystal }-Z}$. The total relative length changes associated to the bainitic transformation: $R L C_{\text {total }-X}^{\text {bainite }}=R L C_{\text {crystal }-X}+R L C_{\text {partition }}^{\text {bainite }}, R L C_{\text {total }-Y}^{\text {bainite }}=R L C_{\text {crystal }-Y}+$ $R L C_{\text {partition }}^{\text {bainite }}$ and $R L C_{\text {total }-Z}^{\text {bainite }}=R L C_{\text {crystal }-Z}+R L C_{\text {partition }}^{\text {bainite }}$.

\section{Results and discussion}

\subsection{Microstructural characterization}

The microstructures analyzed in this work have been thoroughly characterized in previous works [19,22], and only the most relevant characteristics will be highlighted in this manuscript. The microstructures analyzed in this work were obtained by a pure isothermal treatment and three ausforming treatments, see Figure 1. As mentioned, it is important to point out that, in all cases, bainita reaction was the only transformation taking place during the whole treatment and it took place during the isothermal holding. The volume fractions of both ferrite and austenite, $F_{\alpha}$ and $F_{\gamma}$, estimated as explained in Section 2, are included in Table 1. During the isothermal holding at $300{ }^{\circ} \mathrm{C}$, dilatometry measured the relative changes 
in length included in Figure 2(a). Note that such relative changes in length have been measured along the longitudinal direction, i.e. Z. As can be seen, the pure isothermal treatment presents a common dilatometry curve, consisting of an incubation time - during which the signal barely increases - a period of time during which the signal increases very rapidly, as most of the transformation occurs in that period of time, and a last stage where the signal steep until it reaches a plateau [43]. For the ausforming treatments, the signal intensity is lower, being negative for the two conditions where $\mathrm{T}_{\text {def }}$ is the lowest. Moreover, when microstructures were examined on the longitudinal section, it was found that plates were highly aligned at $\pm 45^{\circ}$ when $\mathrm{T}_{\text {def }}$ was either 400 or $300^{\circ} \mathrm{C}$, see Figure 2(d,e). The values of $R L C_{\text {total-Z-exp }}$ after $1 \mathrm{~h}$ are included in Table 1 . In addition, the value of the radial relative length change $\left(R L C_{\text {total-R-exp }}\right)$ after $1 \mathrm{~h}$ for the sample deformed at $\mathrm{T}_{\text {def }}=400{ }^{\circ} \mathrm{C}$, reported in Ref. [22], is also included in Table 1. The work in Ref. [19] showed that the phenomenon of variant selection occurred in all conditions, although it was more pronounced as $\mathrm{T}_{\text {def }}$ decreased. Although these results suggest that variant selection is the reason why dilatometry signals are negative, this hypothesis has not been proved yet. As previously mentioned, in this work, the theory explained in the Section 3 was applied to calculate the relative length changes associated to the bainitic transformation in those four different samples. To do so, XRD and EBSD maps have been used, and in the following sections, the procedure that has been followed to make such calculations will be explained.
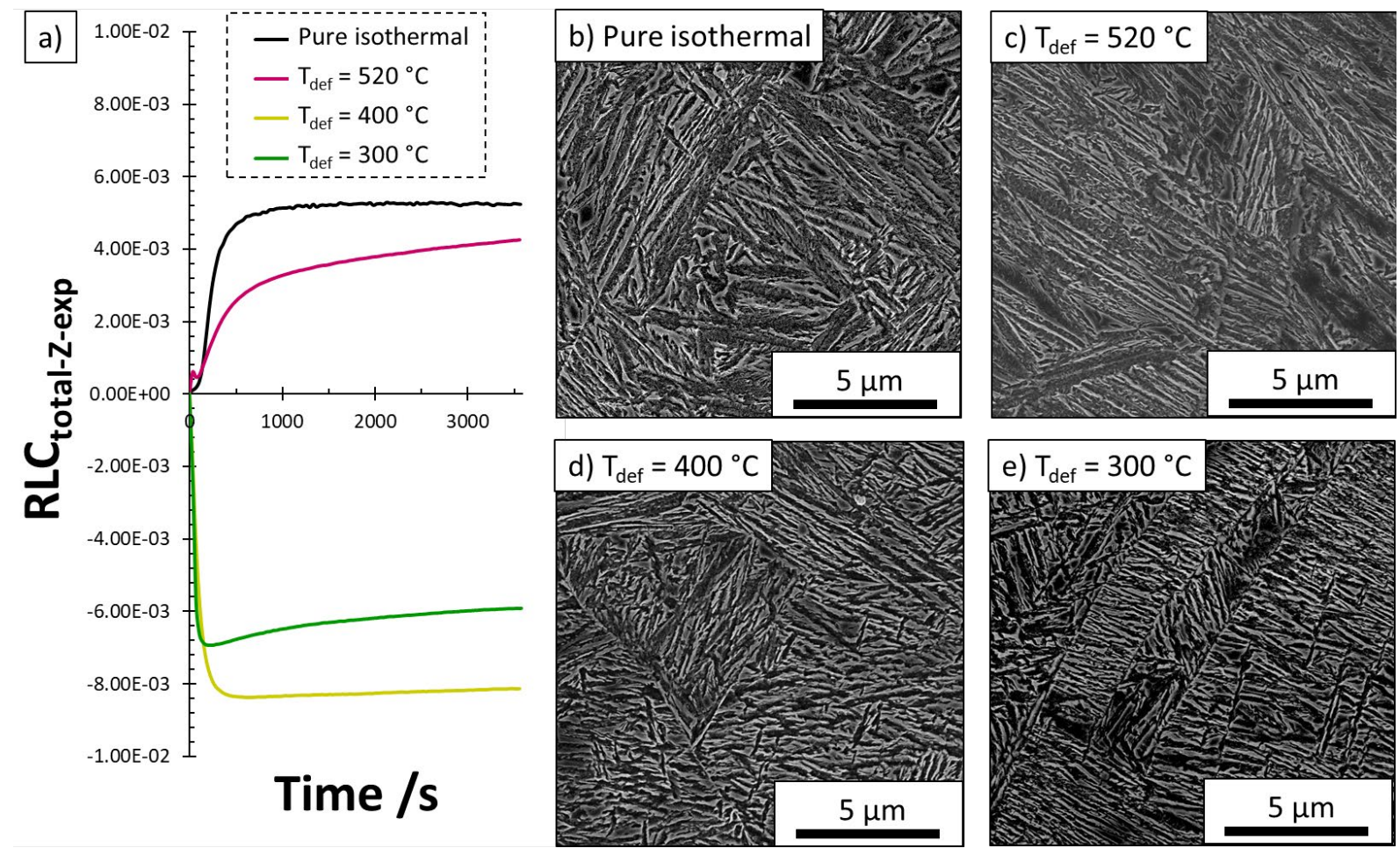

Figure 2. (a) Dilatometry curves obtained during the isothermal holding, at $300{ }^{\circ} \mathrm{C}$, in the treatments studied in this manuscript; (b-e) micrographs of the microstructures obtained by (b) a pure isothermal treatment; (c-e) ausforming treatments with $\mathrm{T}_{\text {def }}$ equal to (d) $520^{\circ} \mathrm{C}$; (d) $400{ }^{\circ} \mathrm{C}$; and (d) $300^{\circ} \mathrm{C}$. All micrographs correspond to the longitudinal section, where the compression was applied (if applied) vertically.

\subsection{Calculation of lattice parameters and volume and length}




\section{changes associated to partition}

For the calculations made in this article, the lattice parameters of $\gamma$ and $\alpha$ at different stages of the transformation had to be calculated. Note that neither of the lattice parameters shown in this work have been experimentally estimated, as one would need in-situ measurements, especially to know the lattice parameters before partition takes place. Subsequently, some of the expressions that were used for such calculations are introduced. The lattice parameter calculation always starts by calculating the lattice parameter at room temperature. While the lattice parameter of $\gamma$ at room temperature (in $\AA$ ), $a_{\gamma, 25}{ }^{\circ} \mathrm{C}$, can be calculated by using the formula from Dyson and Holmes [44], see Equation (40), the lattice parameter of $\alpha$ (in $\AA$ ), $a_{\alpha, 25^{\circ} \mathrm{C}}$, can be calculated by the formula derived by Bhadeshia et al. [21], see Equation (41).

$$
\begin{aligned}
& a_{\gamma, 25{ }^{\circ} \mathrm{C}}=3.5780+0.033 M_{C}+0.0 .00095 M_{M n}+0.0002 M_{N i} \\
&+ 0.0006 M_{C r}+0.022 M_{N}+0.0056 M_{A l} \\
&-0.0004 M_{C o}+0.0015 M_{C u}+0.0031 M_{M o} \\
&+0.0051 M_{N b}+0.0039 M_{T i}+0.0018 M_{V} \\
&+0.0018 M_{W} \\
& a_{\alpha, 25{ }^{\circ} \mathrm{C}}=2.8664+\frac{\left(a_{F e}-0.279 M_{C}\right)^{2}\left(a_{F e}+2.496 M_{C}\right)-a_{F e}^{3}}{3 a_{F e}^{2}} \\
&-0.03 M_{S i}+0.06 M_{M n}+0.07 M_{N i}+0.31 M_{M o} \\
&+ 0.05 M_{C r}+0.096 M_{V}
\end{aligned}
$$

where $M_{i}$ stands for the mole fraction of the element $i$ in the corresponding phase and $a_{F e}$ is the lattice parameter of pure iron $\left(a_{F e}=2.8664 \AA\right)$. The lattice parameters of a phase $p h$ at the transformation temperature $T_{\text {iso }}$ (in ${ }^{\circ} \mathrm{C}$ ) can be calculated by using its thermal expansion coefficient $e_{p h}$, see Equation (42). The thermal expansion coefficients of $\gamma$ and $\alpha$ were taken as $e_{\gamma}=2.065 \cdot 10^{-5}\left({ }^{\circ} \mathrm{C}\right)^{-1}[45]$ and $e_{\alpha}=1.386 \cdot 10^{-5}\left({ }^{\circ} \mathrm{C}\right)^{-1}[46]$.

$$
a_{p h}=a_{p h, 25{ }^{\circ} \mathrm{C}}\left(1+e_{p h}\left(T_{i s o}-25\right)\right)
$$

For the calculation of the lattice parameters before partitioning, $a_{\gamma, B P}$ and $a_{\alpha, B P}$, the bulk chemical composition were used. For the calculation of the lattice parameters after partitioning, $a_{\gamma, A P}$ and $a_{\alpha, A P}$, the molar fraction of each of the elements for each phase was calculated by assuming paraequilibrium conditions, i.e. the weight percent of all the elements but carbon was the same in both phases: the bulk one. Regarding carbon content, it was assumed to be $0.2 \mathrm{wt} . \%$ in $\alpha$, according to the literature [47], whereas the $\gamma$ carbon content was calculated for each case according to the level rule. The lattice parameters and the austenite carbon content after partition, $C_{\gamma, A P}$, calculated according to the level rule by using the volume fractions in Table 1, can be found in Table 2.

Once all these parameters are known, the volume change associated to carbon partition can be calculated by Equation (6) and the relative length changes associated to it can also be calculated by Equation (7). The values of $R V C_{\text {partition }}^{\text {bainite }}$ and $R L C_{\text {partition }}^{\text {bainite }}$ for each condition are included in Table 1. 


\subsection{OR estimation and selection of EBSD area representative of the microstructure}

The calculations were carried out by using data from a previous work [19], in which regions were selected from EBSD maps so that the local textures of the prior austenite (reconstructed by the software by Nyyssönen et al. [28]) and the ferrite were the same than the global XRD textures, find Z-axis Inverse Pole Figures (IPF) of both phases obtained from XRD measurements and from the mentioned EBSD regions in Figure 3. The same regions will be used in this work. Further information can be found in [19]. Note that the non-indexed pixels found in the EBSD maps were filled by MTEX, assuming that the microstructure was fully ferritic for the analysis. The presence of retained austenite will be later considered.

Table 1. For each of the conditions studied in this work: volume fractions of ferrite and austenite $\left(F_{\alpha}\right.$ and $\left.F_{\gamma}\right)$, relative change in length along the longitudinal section obtained by dilatometry after 1 hour isothermal treatment $\left(R L C_{\text {total-Z-exp }}\right)$, relative volume change and relative length change because of carbon partition $\left(R V C_{\text {partition }}^{\text {bainite }}\right.$ and $\left.R L C_{\text {partition }}^{\text {bainite }}\right)$, relative volume change because of the crystal structure change, using $(\gamma \boldsymbol{A} \gamma)$ or $(\gamma \boldsymbol{P} \gamma)$ for its calculation $\left(R V C_{\text {crystal }}\right.$ and $\left.R V C_{\text {crystal-P }}\right)$, relative length change because of the crystal structure change along the $\mathrm{X}, \mathrm{Y}$ and $\mathrm{Z}$ axes $\left(R L C_{\text {crystal }-X}, R L C_{\text {crystal }-Y}\right.$ and $\left.R L C_{\text {crystal-Z }}\right)$, total volume change, using $(\gamma \boldsymbol{A} \gamma)$ or $(\gamma \boldsymbol{P} \gamma)$ for its calculation $\left(R V C_{\text {total }}^{\text {bainite }}\right.$ and $\left.R V C_{\text {total }}^{\text {baini }}-\mathrm{P}\right)$ and total relative length change along the $\mathrm{X}, \mathrm{Y}$ and $\mathrm{Z}$ axes $\left(R L C_{\text {total }-X}^{\text {bainite }}, R L C_{\text {total }-Y}^{\text {bainite }}\right.$ and $\left.R L C_{\text {total }-Z}^{\text {bainite }}\right)$.

\begin{tabular}{|c|c|c|c|c|}
\hline Sample & Pure isothermal & $\mathrm{T}_{\mathrm{def}}=520^{\circ} \mathrm{C}$ & $\mathrm{T}_{\mathrm{def}}=400^{\circ} \mathrm{C}$ & $\mathrm{T}_{\mathrm{def}}=300^{\circ} \mathrm{C}$ \\
\hline$F_{\alpha}( \pm 0.03)$ & 0.86 & 0.79 & 0.76 & 0.74 \\
\hline$F_{\gamma}( \pm 0.03)$ & 0.14 & 0.21 & 0.24 & 0.26 \\
\hline $\begin{array}{c}R L C_{\text {total-Z-exp }} \\
(\operatorname{after} 1 \mathrm{~h})\end{array}$ & $5.249 \cdot 10^{-3}$ & $4.249 \cdot 10^{-3}$ & $-8.141 \cdot 10^{-3}$ & $-5.904 \cdot 10^{-3}$ \\
\hline $\begin{array}{c}R L C_{\text {total-R-exp }} \\
(\text { after } 1 \mathrm{~h})\end{array}$ & - & - & $1.034 \cdot 10^{-2}$ & - \\
\hline$R V C_{\text {partition }}^{\text {bainite }}$ & $3.432 \cdot 10^{-3}$ & $3.157 \cdot 10^{-3}$ & $3.040 \cdot 10^{-3}$ & $2.940 \cdot 10^{-3}$ \\
\hline$R L C_{\text {partition }}^{\text {bainite }}$ & $1.144 \cdot 10^{-3}$ & $1.052 \cdot 10^{-3}$ & $1.013 \cdot 10^{-3}$ & $9.801 \cdot 10^{-4}$ \\
\hline$R V C_{\text {crystal }}$ & $1.103 \cdot 10^{-2}$ & $1.013 \cdot 10^{-2}$ & $9.746 \cdot 10^{-3}$ & $9.489 \cdot 10^{-3}$ \\
\hline$R V C_{\text {crystal-P }}$ & $1.068 \cdot 10^{-2}$ & $9.816 \cdot 10^{-3}$ & $9.444 \cdot 10^{-3}$ & $9.195 \cdot 10^{-3}$ \\
\hline$R L C_{\text {crystal-X }}$ & $3.378 \cdot 10^{-3}$ & $7.118 \cdot 10^{-3}$ & $2.543 \cdot 10^{-2}$ & $2.031 \cdot 10^{-2}$ \\
\hline$R L C_{\text {crystal }-Y}$ & $4.476 \cdot 10^{-3}$ & $4.420 \cdot 10^{-3}$ & $2.207 \cdot 10^{-2}$ & $2.189 \cdot 10^{-2}$ \\
\hline
\end{tabular}




\begin{tabular}{lllll}
$R L C_{\text {crystal-Z }}$ & $2.832 \cdot 10^{-3}$ & $-1.722 \cdot 10^{-3}$ & $-3.805 \cdot 10^{-2}$ & $-3.301 \cdot 10^{-2}$ \\
\hline$R V C_{\text {total }}^{\text {bainite }}$ & $1.446 \cdot 10^{-2}$ & $1.328 \cdot 10^{-2}$ & $1.228 \cdot 10^{-2}$ & $1.243 \cdot 10^{-2}$ \\
$R V C_{\text {total }-P}^{\text {bainite }}$ & $1.411 \cdot 10^{-2}$ & $1.230 \cdot 10^{-2}$ & $1.248 \cdot 10^{-2}$ & $1.214 \cdot 10^{-2}$ \\
$R L C_{\text {total-X }}^{\text {bainite }}$ & $4.522 \cdot 10^{-3}$ & $8.170 \cdot 10^{-3}$ & $2.644 \cdot 10^{-2}$ & $2.129 \cdot 10^{-2}$ \\
$R L C_{\text {total-Y }}^{\text {bainite }}$ & $5.620 \cdot 10^{-3}$ & $5.472 \cdot 10^{-3}$ & $2.301 \cdot 10^{-2}$ & $2.287 \cdot 10^{-2}$ \\
$R L C_{\text {total-Z }}^{\text {bainite }}$ & $3.976 \cdot 10^{-3}$ & $-6.696 \cdot 10^{-4}$ & $-3.704 \cdot 10^{-2}$ & $-3.203 \cdot 10^{-2}$ \\
\hline
\end{tabular}

Table 2. Lattice parameters of ferrite and austenite at the isothermal temperature before and after partition $\left(a_{\gamma, B P}, a_{\alpha, B P}, a_{\gamma, A P}\right.$ and $\left.a_{\alpha, A P}\right)$ and carbon content of austenite after partition, calculated according to the level rule $\left(C_{\gamma, A P}\right)$ by using the volume fractions in Table 1 . They have been calculated as explained in Section 4.1.

$\begin{array}{cccccc}\text { Treatment } & a_{\gamma, B P} / \AA & a_{\alpha, B P} / \AA & a_{\gamma, A P} / \AA & a_{\alpha, A P} / \AA & C_{\gamma, A P} / \mathrm{wt} . \%\end{array}$

Pure isothermal

$\mathrm{T}_{\mathrm{def}}=520^{\circ} \mathrm{C}$

$\mathrm{T}_{\text {def }}=400{ }^{\circ} \mathrm{C}$

$\mathrm{T}_{\text {def }}=300{ }^{\circ} \mathrm{C}$

\subsection{7}

2.8812

3.6500

1.49

3.6375

3.6341

2.8794

1.12

1.01

3.6317

0.94 


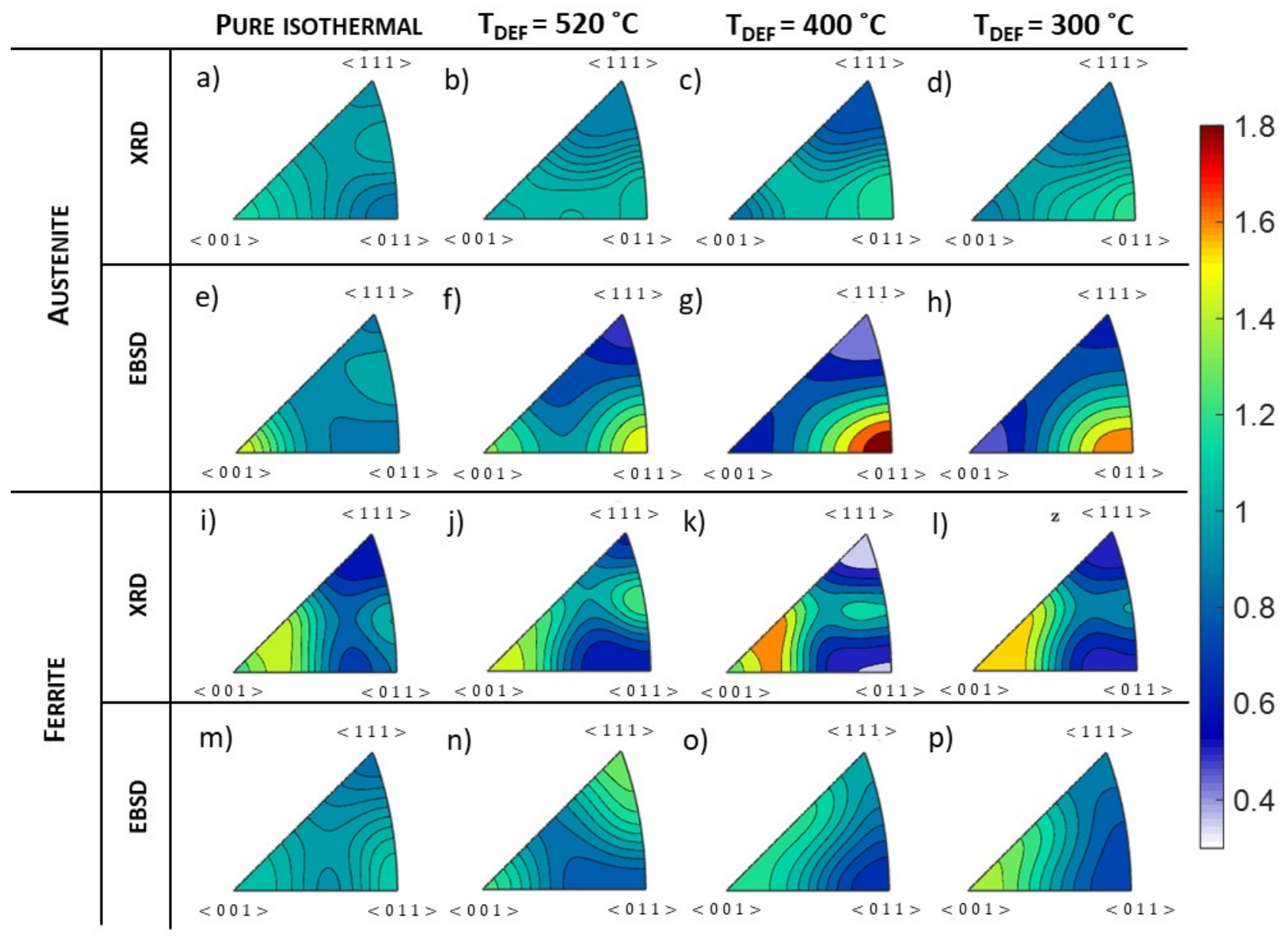

Figure 3. Inverse Pole figures along the compression axis (IPF-Z) obtained by XRD (a-d and i-1) and by selecting some areas of the EBSD maps (e-h and $m-p)$ for austenite (a-h) and for ferrite (i-p). The intensity is expressed in MRD (multiple of random density). Figure modified from [19].

For the analyses, the EBSD regions were divided in ferrite grains, defined as areas with a misorientation lower than $10^{\circ}$. For every ferrite grain, there was a corresponding prior austenite data given by the reconstruction by Nyyssönen et al. [28]. The calculations described in Section 3 were not made pixel by pixel, but ferrite grain by ferrite grain, in order to save computational time. The prior austenite and ferrite orientations of that grain were calculated as an average of the corresponding orientations in that grain.

The orientation relationship $(\gamma J \alpha)$ was estimated from the experimental EBSD maps, as explained in in Ref. [19]: $<0.130 .180 .97>44.41^{\circ}$. Note that, as previously anticipated, the software does not consider tetragonality for its estimation. This OR leads to 24 different variants - and, in turn, rotation matrices. For every ferrite grain, from all possible 24 rotation matrices, the selected rotation matrix $(\gamma J \alpha)$ was the one which, when multiplied by the grain prior austenite orientation, gave the closest orientation to the experimental grain ferrite orientation.

\subsection{Determination of the invariant line, habit plane and dilatational and shear components associated to the}




\section{transformation of the steel of study}

For a given ferrite grain, the theory explained in Section 3.2 can be applied. Some of the most important results given by these calculations will be presented in the following paragraphs.

For the lattice parameters calculated in Section 4.1. the determinant of the deformation gradient tensor is $|(\gamma \boldsymbol{A} \gamma)|=1.0128$. If such tensor is decomposed by the SVD, it can be observed that, as expected, two of the principal deformations are greater than one and equal $\left(\sigma_{A 1}=\sigma_{A 2}=1.1272\right)$, whereas the third of them is less than $1\left(\sigma_{A 3}=0.7971\right)$. Figure 4 shows a black sphere representing the initial volume before any deformation occurs and a red ellipsoid representing the final volume after $(\gamma \boldsymbol{A} \gamma)$ is applied to the position vectors of the initial volume. The thick black line is the IL; it can be observed that such IL intersects both the sphere and the ellipsoid at the intersection of both surfaces. Figure 4 includes a detailed image where the vectors $\left[\gamma ; \boldsymbol{X}_{\exp -I L}\right],\left[\gamma ; \boldsymbol{x}_{\boldsymbol{e x p}-I \boldsymbol{L}}\right]$ and $\left[\gamma ; \boldsymbol{\Delta} \boldsymbol{x}_{\boldsymbol{e x p}-I \boldsymbol{L}}\right]$ are included. As can be observed, $\left[\gamma ; \boldsymbol{x}_{\boldsymbol{e x p}-I \boldsymbol{L}}\right]$ is only slightly displaced with respect to $\left[\gamma ; \boldsymbol{X}_{\boldsymbol{e x p}-I \boldsymbol{L}}\right]$, being $\left\|\left[\gamma ; \boldsymbol{\Delta} \boldsymbol{x}_{\exp -\boldsymbol{I L}}\right]\right\|=5.4817 \cdot 10^{-4}$. Figure 4 also includes the ellipsoid representing $(\gamma \boldsymbol{t} \gamma)$, whose third principal component -obtained by the SVD - $\sigma_{t 3}$ is as long as $\left[\gamma ; \boldsymbol{x}_{\boldsymbol{e x p}-I L}\right]$, i.e. $\sigma_{t 3}=5.4817 \cdot 10^{-4}$, whereas the two first principal components are $\sigma_{t 1}=0.3727$ and $\sigma_{t 2}=0.1176$. Although the displacement of the IL is minimal, it is not zero and this may affect the rest of the calculations, as will be shown. For instance, regarding $(\gamma \boldsymbol{P} \gamma)$ and $(\gamma \boldsymbol{Q} \gamma)$, their determinants are $|(\gamma \boldsymbol{P} \gamma)|=1.0124$ and $|(\gamma \boldsymbol{Q} \gamma)|=1.0004$, while the theory predicted that $|(\gamma \boldsymbol{P} \gamma)|=|(\gamma \boldsymbol{A} \gamma)|$ and $|(\gamma \boldsymbol{Q} \gamma)|=1$. The values of the relative change in volume given by both $(\gamma \boldsymbol{A} \gamma)$ and $(\gamma \boldsymbol{P} \gamma)$, i.e. $R V C_{\text {crystal }}$ and $R V C_{\text {crystal-P }}$, calculated by Equations (13) and (22), are included in Table 1 for each of the conditions studied in this work. The total relative change in volume, calculated by summing up the corresponding $R V C_{\text {crystal }}$ or $R V C_{\text {crystal-P }}$ to $R V C_{\text {partition }}^{\text {bainite }}-R V C_{\text {total }}^{\text {bainite }}$ and $R V C_{\text {total-P }}^{\text {bainite }}$ - are also included. Also, the angle between $(\boldsymbol{q} ; \gamma)$ and $\left[\gamma ; \boldsymbol{x}_{\boldsymbol{e x p}-I \boldsymbol{L}}\right]$ is not $90^{\circ}$, but $87.54^{\circ}$. 


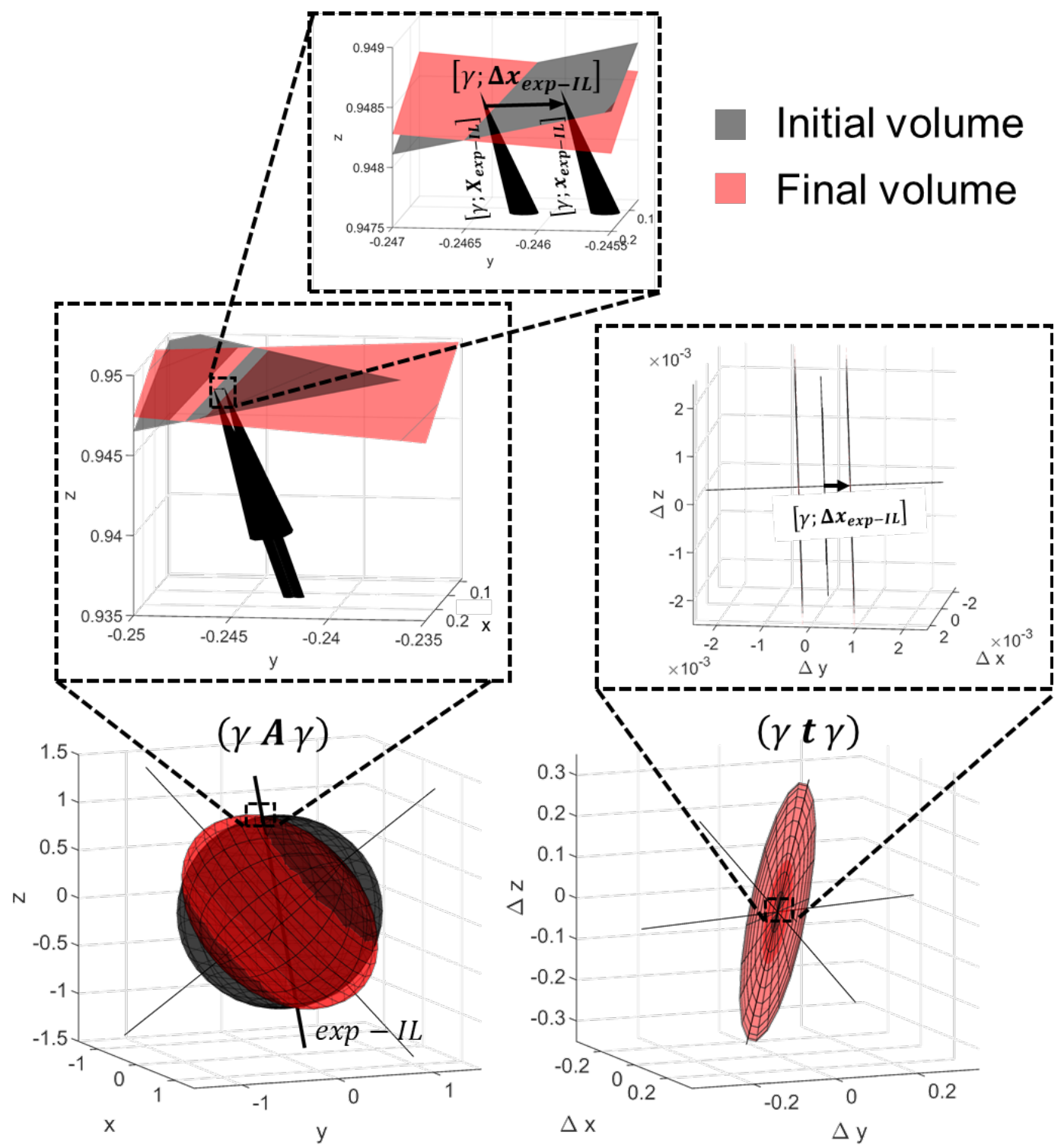

Figure 4. Deformation ellipsoids given by $(\gamma \boldsymbol{A} \gamma)$, where the position vectors of the IL are shown. The displacement ellipsoid given by $(\gamma t \gamma)$ is also shown on the right bottom part.

According to the calculations and assuming that the lattice invariant shear occurs in $\left\{\begin{array}{lll}1 & 0 & 1\end{array}\right\}_{\gamma}<\overline{1} 01>_{\gamma}$ [32], the habit plane in the austenite coordinate system is $(\boldsymbol{p} ; \gamma)=[$ $0.7417,0.6024,0.2948]$. The angle between $(\boldsymbol{p} ; \gamma)$ and $\left[\gamma ; \boldsymbol{x}_{\boldsymbol{e x p}-I \boldsymbol{L}}\right]$ is $89.81^{\circ}$, which proves that the IL is contained in the habit plane. Small deviations could be due to the fact that the IL displacement is not exactly zero. Figure 5 shows an Inverse Pole Figure (IPF) where the habit plane calculated in this work is compared to habit planes that have been estimated by previous authors in martensitic [6,48-55] and bainitic [6,37,48,54,56,57] microstructures. As can be seen, the habit plane calculated in this work is similar to the one estimated by Srinivasan and Wayman in a bainitic microstructure obtained in a high chromium high carbon steel - Fe-8 Cr-1.1C (wt. \%) [48]. Regarding the dilatational and shear components of the macroscopic deformation, they are $\delta=0.0124$ and $s=0.2371$, respectively. Note that these 
values, although slightly low, are of the same order of magnitude than the ones reported in the literature for bainitic transformations [36-40].

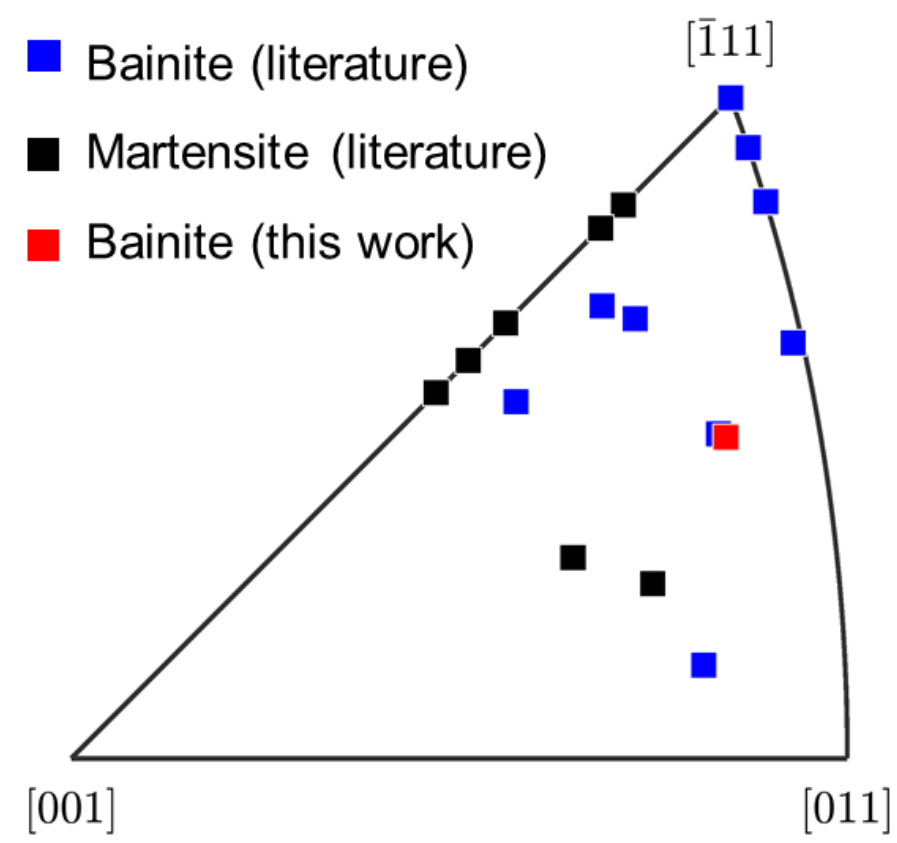

Figure 5. Austenite habit planes in martensitic [6,48-55] and bainitic [6,37,48,54,56,57] microstructures of the literature, compared to the austenite habit plane obtained in this work by the methodology presented.

\subsection{Calculation of relative change lengths along the radial and longitudinal sample axes}

Figure 6 shows the ( $F_{\alpha} \cdot$ Area fraction) weighted histograms of the total relative length change $\left(R L C_{\text {total }}^{\text {bainite }}\right)$ along the longitudinal and the radial directions, calculated as explained in Section 3.2.4. The radial data have been obtained by considering data along both the $X$ and $\mathrm{Y}$ axes. The area was multiplied by $F_{\alpha}$ because only bcc was considered for the EBSD maps and the non-indexed data was filled subsequently by MTEX. As previously mentioned, for the calculations, it was assumed that the bcc lattice occupied the whole microstructure, and results were later weighted by their corresponding bainitic ferrite volume fractions. Those histograms are compared to the one that would be expected for a microstructure with no variant selection, the so-called random distribution. Note that, if no variant selection effects occur, the shear deformations average out at a macroscopic level, the average shear deformation being null and only remaining the isotropic dilatational component [21].

As can be observed, the histograms disagree with the random one in a higher extent as $\mathrm{T}_{\text {def }}$ decreases. Thus, while the histograms are similar regardless of the axis to which they correspond for the pure isothermal treatment and the ausforming treatment performed at 520 ${ }^{\circ} \mathrm{C}$, the longitudinal and the radial histograms shifts towards more negative and more positive values, respectively, as $T_{\text {def }}$ decreases. As expected, the average relative change in length values are also different, as shown in Table 1 . While $R L C_{\text {total-X }}, R L C_{\text {total-Y }}$ and 
$R L C_{\text {total }-Z}$ are of the same order of magnitude and all positive for the pure isothermal condition, $R L C_{\text {total }-Z}$ becomes more negative as $T_{\text {def }}$ decreases. In addition, it can be seen that $R L C_{\text {total }-X}$ and $R L C_{\text {total }-Y}$ increase as $T_{\text {def }}$ decreases, being always positive.

It is clear that the results given by the calculation and those of the longitudinal experimental relative length change ( $R L C_{\text {total-Z-exp }}$ in Table 1$)$, follow the same trends. The longitudinal relative change in length decreases as $\mathrm{T}_{\text {def }}$ does for the two highest values of $\mathrm{T}_{\text {def. }}$ Regarding $\mathrm{T}_{\text {def }}=300{ }^{\circ} \mathrm{C}$, its relative change in length is higher than the one obtained for $\mathrm{T}_{\text {def }}=400{ }^{\circ} \mathrm{C}$, although still negative, see Figure 2(a). Regarding the $R L C_{\text {total-R-exp, as previously }}$ explained, only the value for the sample ausformed at $\mathrm{T}_{\text {def }}=400{ }^{\circ} \mathrm{C}$ is known, see Table 1 . In that case, it can be seen that the value of $R L C_{\text {total-R-exp }}$ is much higher than the value of $R L C_{\text {total-Z-exp }}$ corresponding to the pure isothermal treatment, both of them being positive. This trend is in good agreement with the obtained results.

a) Pure isothermal
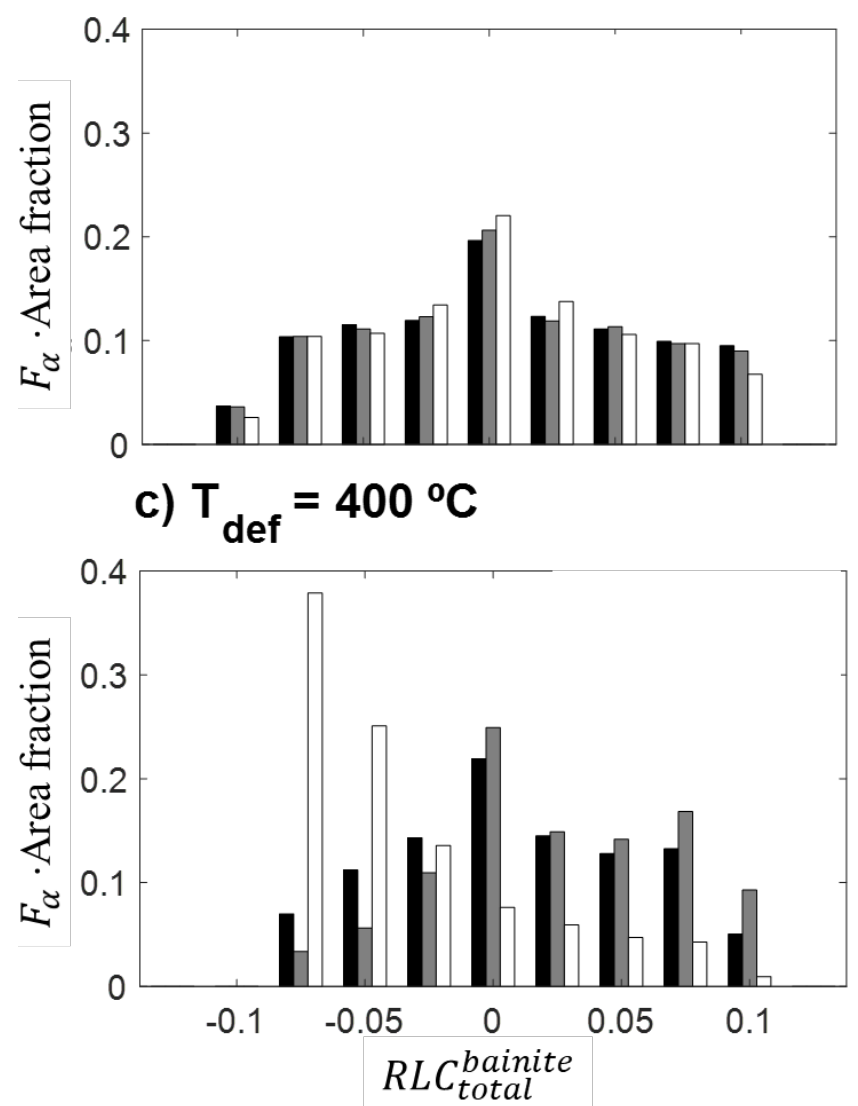

b) $\mathrm{T}_{\mathrm{def}}=520^{\circ} \mathrm{C}$
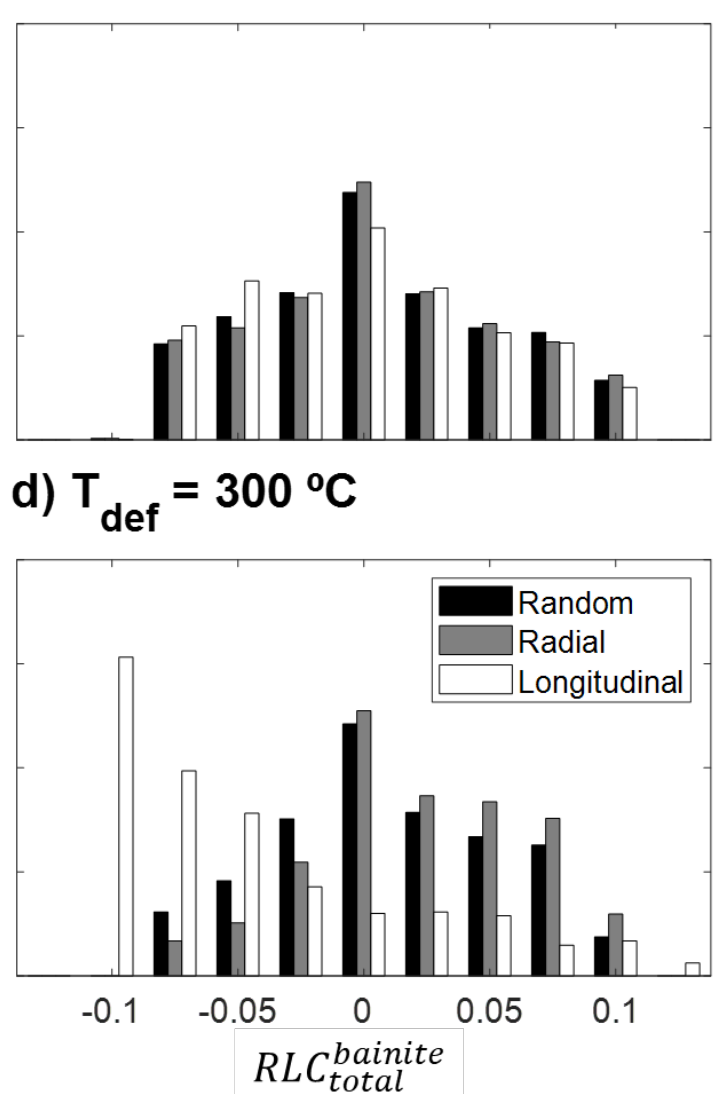

Figure 6. $\left(F_{\alpha} \cdot\right.$ Area fraction) weighted histograms of the total relative length change $\left(R L C_{\text {total }}^{\text {bainite }}\right)$ along the longitudinal and the radial directions, where the radial data have been obtained by considering data along the $\mathrm{X}$ and $\mathrm{Y}$ axes, calculated as explained in Section 3.2.4. Those histograms are compared to the one that would be expected for a microstructure with no variant selection, the so-called random distribution.

Therefore, the negative signals obtained in dilatometry can be explained by using the Phenomenological Theory of Martensite. The discrepancies between the experimental and the theoretical results could be related to a variety of reasons: a) it was assumed that all the 
bainitic ferrite formed and then the partition occurred, which may have led to error; b) the lattice parameters at room temperature were estimated by using expressions which most likely has some associated error and tetragonality was not considered; c) the lattice parameters at higher temperatures were calculated by using thermal expansion coefficients from the literature, which may not be reliable for the given steel; d) the orientation relationship was estimated from EBSD maps by assuming no tetragonality and was considered not to vary for the given steel; the software most likely has an associated error and the OR could also change along the microstructure; e) because of the three previous reasons, the IL displacement was not zero and, although it was rather low, results were slightly affected by it; f) the volume fractions that were used have been estimated by XRD, being their error \pm 0.03 ; g) although areas from EBSD maps were selected so that their texture was similar to the one obtained by $\mathrm{XRD}$, the texture intensity of the EBSD is lower; $h$ ) the phenomenological theory of martensite assumes the Bain correspondence and a lattice invariant strain; these conditions could not be completely met; i) the carbon content in ferrite was assumed based in the literature; j) for the calculation of the relative length changes associated to the partition, it has been assumed that the carbon is homogeneously distributed, whereas carbon usually partitions to Cottrell atmospheres or dislocations, among others; for that reason, results given by the level rule are usually overestimated; and k) dilatometry curves obtained during the thermomechanical treatments may not be accurate because the changes in length were measured with silicon nitride push-rods, which have a high thermal expansion coefficient.

\subsection{Extrapolation of the methodology for martensitic structures}

The methodology explained in the present work could be applied to martensitic transformations, instead of to bainitic ones by making some modifications that will be subsequently enumerated.

Firstly, for martensitic transformations, the composition of both phases is the same - the bulk composition - at any point of the transformation. Therefore, in the case of martensitic transformations, the total relative volume change ( $R V C_{\text {total }}^{\text {martensite }}$ ) associated to the transformation is equal to the relative volume change associated to the change of crystallographic structure $\left(R V C_{\text {crystal }}\right)$, as shown in Equation (43). This means that the sections which focused on the calculation of volume and length changes due to the partition of carbon should not be applied for martensitic transformations.

$$
R V C_{\text {total }}^{\text {martensite }}=R V C_{\text {crystal }}
$$

Also, because martensite is formed during cooling, in this case it would be compulsory to carry out the calculation incrementally and the martensite start (Ms) and martensite end (Mf) temperatures should be known. For every temperature step in the range Ms-Mf, it should be considered that a certain amount of martensite is transformed - this amount of martensite could be considered to be fixed or to change as the transformation progresses -and the lattice parameters of austenite and ferrite should be calculated at that temperature by Equation (42). Once those two data were known, the relative volume and length changes associated to the martensitic transformation in every incremental step could be calculated. 


\section{Conclusion}

In this work, a methodology to calculate the relative length changes associated to the bainitic transformation along the $\mathrm{X}, \mathrm{Y}$ and $\mathrm{Z}$ axes has been proposed to explain the reason why variant selection leads to anisotropic dilatometry signals in some instances. Although the methodology has been applied to bainitic microstructures, some hints about its extrapolation to martensitic structures have been given.

The mentioned methodology has been tested with experiments from previous works, which presented negative longitudinal dilatometry signals during the bainitic transformation in some cases and pronounced variant selection phenomena. It has been proved that, even though the dilatometry signal is not the same than the one calculated, the trends are kept. Several reasons of the discrepancies between the experimental and the theoretical results have been given.

Also, the habit plane has been calculated, being in good agreement with a habit plane experimentally estimated in the literature.

\section{Acknowledgments}

The authors gratefully acknowledge the support for this work by the European Research Fund for Coal and Steel under the Contract RFCS-2015-709607. This work was also partially supported by the European Research Fund for Coal and Steel under the Contract RFCS-2019899251. The authors acknowledge Sidenor for providing them with the material, the support of the X-Ray Diffraction lab (CENIM), the Metallography and Phase Transformations labs (CENIM) and the Electron Microscopy Service facility (Polytechnic School of Valencia). Lucia Morales-Rivas acknowledges funding support from the German Research Foundation (DFG), project number: 411091845. Adriana Eres-Castellanos acknowledges the German Academic Exchange Service (DAAD) for the support received under the funding program Research Grants - Short-Term Grants (57440917), personal ref 91710971.

\section{Appendix A: Notation}

The following notation rules have been followed in this work:

- All matrices and vectors are bolded, whereas scalars are not.

- The matrix representation of a column vector $\boldsymbol{u}$ in a coordinate system $A$ is $[A ; \boldsymbol{u}]$.

- The matrix representation of a row vector $\boldsymbol{u}$ in a coordinate system $A$ is $(\boldsymbol{u} ; A)$.

- Matrices in this work are tensors that can represent operations in a same coordinate system, such as rotations or deformations, or operations of change of basis. Anyhow, a tensor $T$ which represents an operation in a coordinate system $A$ will be named $(A \boldsymbol{T} A)$, whereas a tensor T representing a change of basis from $A$ to $B$ will be named $(B \boldsymbol{T} A)$.

- In this work, some continuum mechanics were applied. In this sense, vectors usually represent direction, plane or position vectors before or after a deformation has been applied. To distinguish, it was decided to use capital letters for all the vectors that represent the initial configuration, whereas the final configuration vector names are always little letters. Regarding tensors, their names are capitalized if they are material tensors, i.e. they are applied to initial position vectors, whereas they are not capitalized if they are spatial tensors, i.e. they are applied to final position vectors. 


\section{Appendix B: Analytical determination of the invariant line}

In this appendix, it will be explained how to analytically calculate the IL, for which some equations of the main text will be referred to. As mentioned, the IL is defined by an infinite number of position vectors, all of them pointing the same direction, and whose spatial displacement vector $\left[\gamma ; \boldsymbol{\Delta} \boldsymbol{x}_{\boldsymbol{I L}}\right]=\left[\begin{array}{lll}0 & 0 & 0\end{array}\right]^{T}$.

To calculate it, $[\gamma ; \boldsymbol{x}]$ and $[\gamma ; \boldsymbol{\Delta x}]$ must be substituted for $\left[x_{I L} y_{I L} z_{I L}\right]^{T}$ and $\left[\gamma ; \boldsymbol{\Delta} \boldsymbol{x}_{\boldsymbol{I L}}\right]$ in Equation (17) and $(\gamma t \gamma)$ must be substituted for the tensor in Equation (18) in Equation (17), obtaining the next equation system:

$$
\left\{\begin{array}{l}
t_{11} x_{I L}+t_{12} y_{I L}+t_{13} z_{I L}=0 \\
t_{21} x_{I L}+t_{22} y_{I L}+t_{23} z_{I L}=0 \\
t_{31} x_{I L}+t_{32} y_{I L}+t_{33} z_{I L}=0
\end{array}\right.
$$

This equation system is homogeneous, as all its constant terms are zero. Because this system is homogeneous, it always has at least one solution, which is the zero (or trivial) solution: $\left[\begin{array}{lll}x_{I L} & y_{I L} & z_{I L}\end{array}\right]^{T}=\left[\begin{array}{lll}0 & 0 & 0\end{array}\right]^{T}$. However, the trivial solution has no physical meaning in this case, as it is the position vector of only one point, not a line. In order for the system to have nontrivial solutions, the rank of $(\gamma \boldsymbol{t} \gamma)$ must be lower than 3, i.e. $|(\gamma \boldsymbol{t} \gamma)|=0$. Moreover, if the wanted non-trivial solutions must be located along only one line, the rank of $(\gamma t \gamma)$ must be 2. Note that if such rank was 1 , the solution would be a plane. Assuming that the rank of $(\gamma t \gamma)$ is 2, the solution of the indeterminate equation system in Equation (B1) is given in Equation (B2):

$$
\left\{\begin{array}{c}
x_{I L}=\lambda \\
y_{I L}=\frac{\left(t_{13} t_{21}-t_{11} t_{23}\right)}{\left(t_{12} t_{23}-t_{13} t_{22}\right)} \lambda \\
z_{I L}=\frac{\left(t_{11} t_{22}-t_{12} t_{21}\right)}{\left(t_{12} t_{23}-t_{13} t_{22}\right)} \lambda
\end{array} \quad \forall \lambda \in \mathbb{R}\right.
$$

If $\left[\gamma ; \boldsymbol{x}_{\boldsymbol{I}}\right]$ is forced to be a unit vector, the first equation in Equation (B2) can be removed and the new system, shown in Equation (B3), can be solved as it has only one solution which is non-trivial.

$$
\left\{\begin{array}{c}
x_{I L}=\sqrt{1-y_{I L}^{2}-z_{I L}^{2}} \\
y_{I L}=\frac{\left(t_{13} t_{21}-t_{11} t_{23}\right)}{\left(t_{12} t_{23}-t_{13} t_{22}\right)} x_{I L} \\
z_{I L}=\frac{\left(t_{11} t_{22}-t_{12} t_{21}\right)}{\left(t_{12} t_{23}-t_{13} t_{22}\right)} x_{I L}
\end{array}\right.
$$

Therefore, the unit vector along the invariant line, $\left[\gamma ; \boldsymbol{x}_{\boldsymbol{I L}}\right]$, obtained as previously explained, is: 


$$
=\frac{\left[\gamma ; \boldsymbol{x}_{I L}\right]}{\left(t_{11}^{2} t_{22}^{2}+t_{11}^{2} t_{23}^{2}-2 t_{11} t_{12} t_{21} t_{22}-2 t_{11} t_{13} t_{21} t_{23}+t_{12}^{2} t_{21}^{2}+t_{12}^{2} * t 23^{2}-2 t_{12} t_{13} t_{22} t_{23}+t_{13}^{2} * t_{21}^{2}+t_{13}^{2} * t_{22}^{2}\right)^{\frac{1}{2}}} .
$$

$$
\cdot\left[\begin{array}{lll}
t_{12} t_{23}-t_{13} t_{22} & t_{13} t_{21}-t_{11} t_{23} & t_{11} t_{22}-t_{12} t_{21}
\end{array}\right]^{T}
$$

Note that the solution would be identic in case the second or the third equations had been substituted by $x_{I L}=\sqrt{1-y_{I L}^{2}-z_{I L}^{2}}$, instead of the first one.

In the practice, when experimental results are used, it is very difficult to work with matrices $(\gamma \boldsymbol{t} \gamma)$ whose rank is 2. The equations in the system found in Equation (18) are most of the times independent and the different selections of the equation to be substituted by $x_{I L}=$ $\sqrt{1-y_{I L}^{2}-z_{I L}^{2}}$ could lead to slightly different results for $\left[\gamma ; \boldsymbol{x}_{I L}\right]$.

\section{Appendix C: Singular Value Decomposition}

The Singular Value Decomposition (SVD) is a very common factorization in linear algebra, valid for any real matrix $[58,59]$. Although it can be applied to matrices of any size, in this work, only the SVD of squared matrices will be explained for a better understanding. Let $(\beta \boldsymbol{M} \beta)$ be a $(m \times m)$ matrix, in a basis $\beta$. Then, its SVD is shown in Equation $(\mathrm{C} 1)$ :

$$
(\beta \boldsymbol{M} \beta)=(\beta \boldsymbol{U} \beta)(\beta \boldsymbol{D} \beta)(\beta \boldsymbol{V} \beta)^{T}
$$

where $(\beta \boldsymbol{U} \beta)$ and $(\beta \boldsymbol{V} \beta)$ are $(m \times m)$ orthonormal matrices, i.e. square matrices whose transpose equals their corresponding inverse, and $(\beta \boldsymbol{D} \beta)$ is a $(m \times m)$ diagonal matrix, whose diagonal entries are known as singular values, $\sigma_{i} \forall i=1 \ldots m$. The decomposition is typically carried out so that $\sigma_{1}>\sigma_{2}>\cdots>\sigma_{n}$.

Regarding the geometrical meaning of those matrices, imagine that $\beta$ is a basis of $\mathbb{R}^{2}$, i.e. we work in a 2 -dimensional space. If $(\beta \boldsymbol{M} \beta)$ is interpreted as a transformation matrix so that $[\beta ; \boldsymbol{x}]=(\beta \boldsymbol{M} \beta)[\beta ; \boldsymbol{X}]$, the matrices $=(\beta \boldsymbol{U} \beta)$ and $(\beta \boldsymbol{V} \beta)^{T}$ represent rotations and $(\beta \boldsymbol{D} \beta)$ represents a scaling transformation. The meaning of this transformation can be better understood if the position vector $[\beta ; \boldsymbol{X}]$ is made to be a unit vector and considered to belong to a set of position vectors forming a circle, see Figure C.1. First $[\beta ; \boldsymbol{X}]$ should be rotated by using $(\beta \boldsymbol{V} \beta)^{T}$ so that the axes along which the dimension change must be applied are aligned with the basis $\beta$ vectors $-\left[\beta ; \boldsymbol{e}_{1}\right]$ and $\left[\beta ; \boldsymbol{e}_{2}\right]$. The result of such rotation is denoted as $\left[\beta ; \boldsymbol{x}_{\boldsymbol{V}^{T}}\right]=(\beta \boldsymbol{V} \beta)^{T}[\beta ; \boldsymbol{X}]$. Subsequently, $\left[\beta ; \boldsymbol{x}_{\boldsymbol{V}^{T}}\right]$ is scaled by applying $(\beta \boldsymbol{D} \beta)$ - note that this scaling implies that the components of the vector $\left[\beta ; \boldsymbol{x}_{V^{T}}\right]$ are multiplied by $\sigma_{1}$ and $\sigma_{2}$, respectively. The resulting vector is then $\left[\beta ; \boldsymbol{x}_{\boldsymbol{D} \boldsymbol{V}^{\boldsymbol{T}}}\right]=$ $(\beta \boldsymbol{D} \beta)\left[\beta ; \boldsymbol{x}_{\boldsymbol{V}^{\boldsymbol{T}}}\right]$. Finally, such vector $\left[\beta ; \boldsymbol{x}_{\boldsymbol{D} \boldsymbol{V}^{T}}\right]$ is rotated with the matrix $(\beta \boldsymbol{U} \beta)$, giving

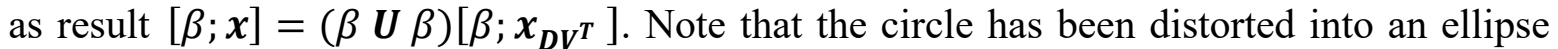
and that the major and minor axes of this ellipse are $\sigma_{1}\left[\beta ; \boldsymbol{u}_{1}\right]$ and $\sigma_{2}\left[\beta ; \boldsymbol{u}_{2}\right]$, whose directions are $\left[\beta ; \boldsymbol{u}_{1}\right]$ and $\left[\beta ; \boldsymbol{u}_{2}\right]$, the columns of the matrix $(\beta \boldsymbol{U} \beta)$, and their modulus are the values $\sigma_{1}$ and $\sigma_{2}$. For further information on the SVD, one can refer to Ref. [58,59]. 

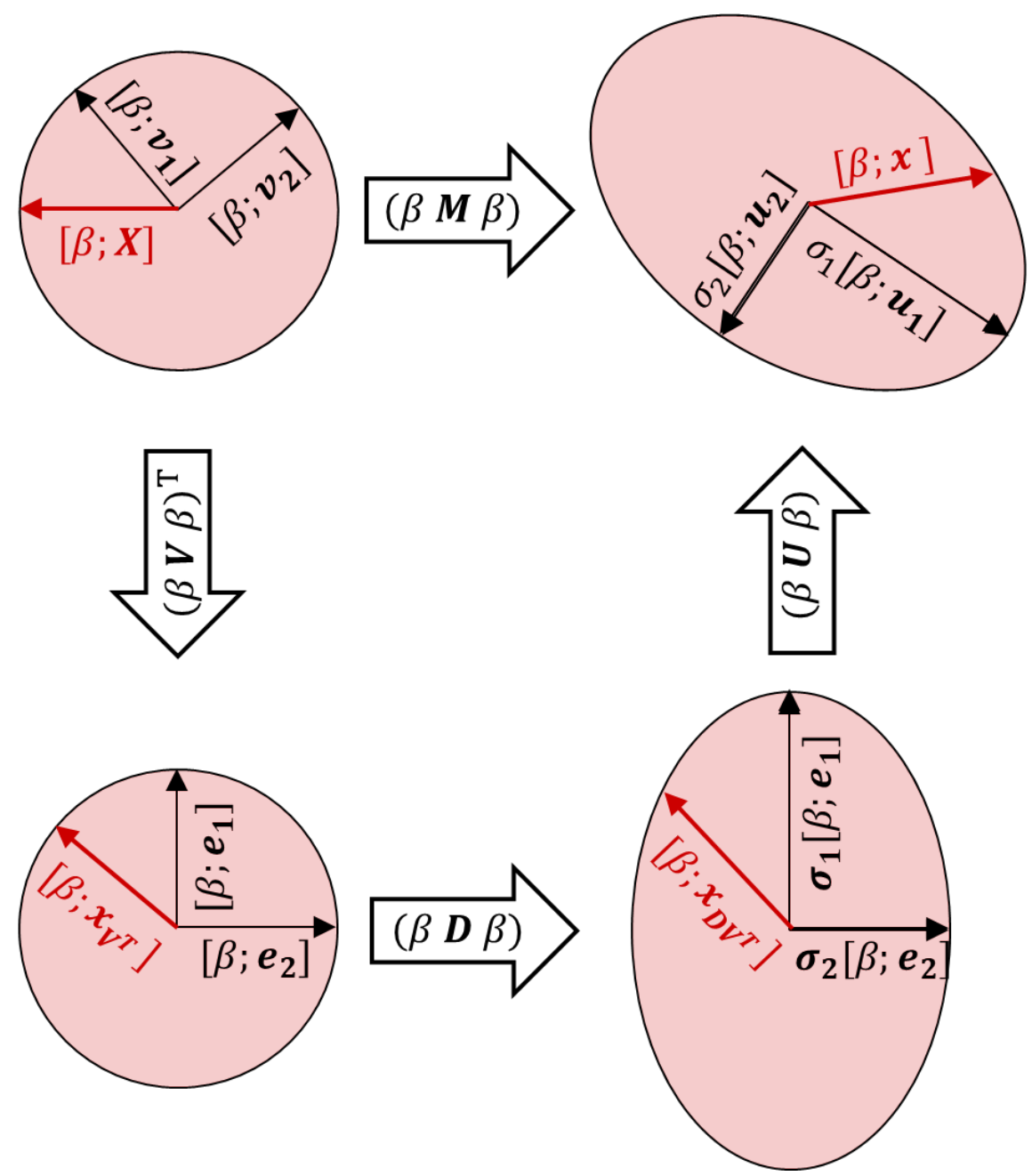

Figure C.1. Illustration of the SVD. Variables used in the figure are described in the main text.

\section{References}

[1] H.K.D.H. Bhadeshia, R.W.K. Honeycombe, Steels: Microstructure And Properties, Butterworths-Heinemann (Elsevier), 2006. http://books.google.es/books?id=6MtuBqok43MC.

[2] E.C. Bain, N.Y. Dunkirk, The nature of martensite, Trans. Metall. Soc. AIME. 70 (1924) 25-47.

[3] G. Wassermann, Ueber den Mechanismus der [alpha]-[gamma]-Umwandlung des Eisens, Verlag Stahleisen, 1935.

[4] Z. Nishiyama, X-ray investigation of the mechanism of the transformation from face centered cubic lattice to body centered cubic, Sci. Rep. Tohoku Imp. Univ. 23 (1934) 637-664.

http://sfx.bibliotecas.csic.es/sfx_local?sid=BMC\&genre=article\&volume=23\&spage $=637 \&$ epage $=664 \&$ date $=1934 \&$ atitle $=X$-ray investigation of the mechanism of the transformation from face centered cubic lattice to body centered cubic\&title $=$ Scientific Report Tohoku.

[5] G. Kurdjumow, G. Sachs, Über den Mechanismus der Stahlhärtung, Zeitschrift fur Phys. . 64 (1930) 325-343. https://doi.org/10.1007/bf01397346. 
[6] A.B. Greninger, A.R. Troiano, The mechanism of Martensite formation, JOM. 1 (1949) 590-598. https://doi.org/10.1007/BF03398900.

[7] Y. He, S. Godet, J.J. Jonas, Observations of the Gibeon meteorite and the inverse Greninger-Troiano orientation relationship, J. Appl. Crystallogr. 39 (2006) 72-81.

[8] W. Pitsch, The martensite transformation in thin foils of iron-nitrogen alloys, Philos. Mag. 4 (1959) 577-584.

[9] T. Furuhara, K. Kikumoto, H. Saito, T. Sekine, T. Ogawa, S. Morito, T. Maki, Phase transformation from fine-grained austenite, ISIJ Int. 48 (2008) 1038-1045.

[10] C. Celada-Casero, J. Sietsma, M.J. Santofimia, The role of the austenite grain size in the martensitic transformation in low carbon steels, Mater. Des. 167 (2019) 107625.

[11] S. Takaki, K. Fukunaga, J. Syarif, T. Tsuchiyama, Effect of grain refinement on thermal stability of metastable austenitic steel, Mater. Trans. 45 (2004) 2245-2251.

[12] J. Hidalgo, M.J. Santofimia, Effect of prior austenite grain size refinement by thermal cycling on the microstructural features of as-quenched lath martensite, Metall. Mater. Trans. A. 47 (2016) 5288-5301.

[13] H.K.D.. Bhadeshia, Bainite in steels: theory and practice, CRC Press, 2019.

[14] S. Kundu, A.K. Verma, V. Sharma, Quantitative analysis of variant selection for displacive transformations under stress, Metall. Mater. Trans. A. 43 (2012) 25522565.

[15] J.R. Patel, M. Cohen, Criterion for the action of applied stress in the martensitic transformation, Acta Metall. 1 (1953) 531-538. https://doi.org/10.1016/00016160(53)90083-2.

[16] W. Gong, Y. Tomota, Y. Adachi, A.M. Paradowska, J.F. Kelleher, S.Y. Zhang, Effects of ausforming temperature on bainite transformation, microstructure and variant selection in nanobainite steel, Acta Mater. 61 (2013) 4142-4154.

[17] W. Gong, Y. Tomota, M.S. Koo, Y. Adachi, Effect of ausforming on nanobainite steel, Scr. Mater. 63 (2010) 819-822. http://www.scopus.com/inward/record.url?eid=2s2.0-77955510496\&partnerID=40\&md5=7e3a42925618dc3376a251a01a21 ef96.

[18] N.F. Viana, C. dos Santos Nunes, H.F.G. de Abreu, The variant selection in the transformation from austenite to martensite in samples of maraging-350 steel, J. Mater. Res. Technol. 2 (2013) 298-302.

[19] A. Eres-Castellanos, L. Morales-Rivas, J.A. Jimenez, F.G. Caballero, C. GarciaMateo, Effect of ausforming on the macro and micro-texture of bainitic microstructures, Under Rev. Acta Mater. (2020).

[20] T. Furuhara, H. Kawata, S. Morito, T. Maki, Crystallography of upper bainite in FeNi-C alloys, Mater. Sci. Eng. A. $431 \quad$ (2006) 228-236. https://doi.org/10.1016/j.msea.2006.06.032.

[21] H.K.D.H. Bhadeshia, S.A. David, J.M. Vitek, R.W. Reed, Stress induced transformation to bainite in Fe-Cr-Mo-C pressure vessel steel, Mater. Sci. Technol. . 7 (1991) 686-698. http://www.scopus.com/inward/record.url?eid=2-s2.00026206981\&partnerID=40\&md5=b270fdbf73171e8d37e2b88a0ca8553a.

[22] A. Eres-Castellanos, L. Morales-Rivas, A. Latz, F.G.F.G.F.G. Caballero, C. GarciaMateo, Effect of ausforming on the anisotropy of low temperature bainitic $\begin{array}{lllll}\text { transformation, } & \text { Mater. } & \text { Charact. } & 145 & \text { (2018) 371-380. }\end{array}$ https://doi.org/10.1016/j.matchar.2018.08.062.

[23] S.B. Singh, H.K.D.H. Bhadeshia, Quantitative evidence for mechanical stabilisation of bainite, Mater. Sci. Technol. . 12 (1996) 610-612. 
[24] J. He, J. Du, W. Zhang, C. Zhang, Z.-G. Yang, H. Chen, Abnormal Anisotropic Dilatation During Bainitic Transformation of Ausformed Austenite, Metall. Mater. Trans. A. 50 (2019) 540-546.

[25] A. Eres-Castellanos, F.G. Caballero, C. Garcia-Mateo, Stress or strain induced martensitic and bainitic transformations during ausforming processes, Acta Mater. 189 (2020) 60-72. https://doi.org/10.1016/j.actamat.2020.03.002.

[26] H.-J. Bunge, Texture analysis in materials science: mathematical methods, Elsevier, 2013.

[27] F. Bachmann, R. Hielscher, H. Schaeben, Texture analysis with MTEX-free and open source software toolbox, in: Solid State Phenom., Trans Tech Publ, 2010: pp. 63-68.

[28] T. Nyyssönen, P. Peura, V.T. Kuokkala, Crystallography, Morphology, and Martensite Transformation of Prior Austenite in Intercritically Annealed High-Aluminum Steel, Metall. Mater. Trans. A Phys. Metall. Mater. Sci. (2018). https://doi.org/10.1007/s11661-018-4904-9.

[29] Y. Xu, G. Xu, X. Mao, G. Zhao, S. Bao, Method to evaluate the kinetics of bainite transformation in low-temperature nanobainitic steel using thermal dilatation curve analysis, Metals (Basel). 7 (2017) 330.

[30] T. Ko, S.A. Cottrell, The formation of bainite, J. Iron Steel Inst. 172 (1952) 307-313.

[31] J.W. Christian, A.G. Crocker, Dislocations and lattice transformations, Dislocations in Solids. 3 (1980) 165-249.

[32] H.K.D.H. Bhadeshia, Worked Examples in the Geometry of Crystals, 1991. https://doi.org/10.1524/zkri.1991.195.1-2.155.

[33] E.S. Machlin, M. Cohen, Burst phenomenon in the martensitic transformation, JOM. 3 (1951) 746-754.

[34] G.B. Olson, M. Cohen, Theory of martensitic nucleation: a current assessment, in: Unkn. Host Publ. Title, Metallurgical Soc of AIME, 1982: pp. 1145-1164.

[35] H.K.D.H. Bhadeshia, Geometry of crystals, polycrystals, and phase transformations, CRC press, 2017.

[36] D.P. Dunne, C.M. Wayman, The crystallography of ferrous martensites, Metall. Trans. (1971). https://doi.org/10.1007/BF02814872.

[37] B.P.J. Sandvik, The Bainite reaction in Fe-Si-C Alloys: The primary stage, Metall. Trans. A. 13 (1982) 777-787. https://doi.org/10.1007/bf02642391.

[38] P. Yan, H.K.D.H. Bhadeshia, Mechanism and kinetics of solid-state transformation in high-temperature processed linepipe steel, in: Metall. Mater. Trans. A Phys. Metall. Mater. Sci., 2013. https://doi.org/10.1007/s11661-013-1907-4.

[39] E. Swallow, H.K.D.H. Bhadeshia, High resolution observations of displacements caused by bainitic transformation, Mater. Sci. Technol. . 12 (1996) 121-125. http://www.scopus.com/inward/record.url?eid=2-s2.00000228603\&partnerID=40\&md5=92f156e962e2f7bce1c800cf0d54fa2c.

[40] M.J. Peet, H.K.D.H. Bhadeshia, Surface Relief Due to Bainite Transformation at $473 \mathrm{~K}\left(200^{\circ} \mathrm{C}\right)$, Metall. Mater. Trans. A Phys. Metall. Mater. Sci. . 42 (2011) 33443348 . $\quad$ http://www.scopus.com/inward/record.url?eid=2-s2.079958279554\&partnerID=40\&md5=bdc68e0b0ebc2276fad0cb4b9ec7df7a.

[41] H.K.D.H. Bhadeshia, A.R. Waugh, Bainite: An atom-probe study of the incomplete reaction phenomenon, Acta Metall. $30 \quad$ (1982) 775-784. http://www.scopus.com/inward/record.url?eid=2-s2.0-

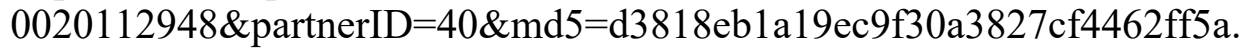


[42] L.C. Chang, H.K.D.H. Bhadeshia, Carbon Content Of Austenite In Isothermally Transformed 300m Steel, Mater. Sci. Eng. A-STRUCTURAL Mater. Prop. Microstruct. Process. 184 (1994) L17-L19.

[43] H.K.D.H. Bhadeshia, Thermodynamic analysis of isothermal transformation diagrams, Met. Sci. 16 (1982) 159-165.

[44] D.J. Dyson, B. Holmes, Effect Of Alloying Additions On Lattice Parameter Of Austenite, J. Iron Steel Inst. 208 (1970) 469-474.

[45] A.S. Podder, I. Lonardelli, A. Molinari, H.K.D.H. Bhadeshia, Thermal stability of retained austenite in bainitic steel: An in situ study, Proc. R. Soc. A Math. Phys. Eng. Sci. $\quad 467 \quad$ (2011) 3141-3156. https://doi.org/10.1098/rspa.2011.021210.2355/isijinternational.42.1059; Bhadeshia, H.K.D.H., 52nd Hatfleld Memorial Lecture large chunks of very strong steel (2005) Materials Science and Technology, 21 (11), pp. 1293-1302. , DOI 10.1179/174328405X63999; Bhadeshia, H.K.D.H., Steels for rails (2007) Encyclopedia of Materials Science, pp. 1-7. , Oxford, UK: Pergamon Press/Elsevier Science; Bhadeshia, H.K.D.H., Nanostructured bainite (2010) Proc. R. Soc. A, 466, pp. 3-18. , doi:10.1098/rspa.2009.0407; Bhadeshia,.

[46] S.S. Babu, E.D. Specht, S.A. David, E. Karapetrova, P. Zschack, M. Peet, H. Bhadeshia, In-situ observations of lattice parameter fluctuations in austenite and transformation to bainite, Metall. Mater. Trans. A-PHYSICAL Metall. Mater. Sci. 36A (2005) 3281-3289.

[47] W.T. Reynolds Jr, S.S. Brenner, H.I. Aaronson, An FIM/AP study of the Mo concentration within ferrite/austenite interfaces in an Fe-0.88 at\% C-1.06 at\% Mo alloy, Scr. Metall. 22 (1988) 1343-1348.

[48] G.R. Srinivasan, C.M. Wayman, Transmission electron microscope study of the bainite transformation in iron-chromium-carbon alloys, Acta Metall. 16 (1968) 609-620. https://doi.org/10.1016/0001-6160(68)90135-1.

[49] E. Pereloma, D. V Edmonds, Phase transformations in steels: Diffusionless transformations, high strength steels, modelling and advanced analytical techniques, Elsevier, 2012.

[50] Y. Inokuti, B. Cantor, The microstructure and kinetics of martensite transformations in splat-quenched Fe and Fe-Ni alloys-I. Pure Fe, Acta Metall. 30 (1982) 343-356.

[51] P.M. Kelly, A. Jostsons, R.G. Blake, The orientation relationship between lath martensite and austenite in low carbon, low alloy steels, Acta Metall. Mater. 38 (1990) 1075-1081. https://doi.org/10.1016/0956-7151(90)90180-O.

[52] P.M. Kelly, The martensite transformation in steels with low stacking fault energy, Acta Metall. 13 (1965) 635-646. https://doi.org/10.1016/0001-6160(65)90126-4.

[53] A. Van Gent, F. Van Doorn, E. Mittemeijer, Crystallography and tempering behavior of Fe-N Martensite, Metall. Trans. A. 16 (1985). http://www.springerlink.com/index/DP56441V3543155H.pdf.

[54] A.B. Greninger, A.R. Troiano, Crystallography of austenite decomposition, Trans. AIME. 140 (1940) 307-336.

[55] G. V Smith, R.F. Mehl, Lattice relationships in decomposition of austenite to pearlite, bainite and martensite, Trans. AIME. 150 (1942) 14.

[56] H. Beladi, V. Tari, I.B. Timokhina, P. Cizek, G.S. Rohrer, A.D. Rollett, P.D. Hodgson, On the crystallographic characteristics of nanobainitic steel, Acta Mater. (n.d.). https://doi.org/http://dx.doi.org/10.1016/j.actamat.2017.01.058. 
[57] S. Hoekstra, H.M.M. Van Der Lelie, C.A. Verbraak, A general method of habit plane determination in bainitic steels-II. Habit plane determination of the bainite, Acta Metall. 26 (1978) 1517-1527. https://doi.org/10.1016/0001-6160(78)90062-7.

[58] N. Lord, G.H. Golub, C.F. Van Loan, Matrix Computations, Math. Gaz. (1999). https://doi.org/10.2307/3621013.

[59] J. Raghavendar, V. Dharmaiah, Geometrical Interpretation of Singular Value Decomposition(Svd) \& Applications of SVD, Int. J. Sci. Innov. Math. Res. 5 (2017) 23-26. https://doi.org/10.20431/2347-3142.0504004. 